\title{
SUBELLIPTIC OPERATORS ON LIE GROUPS: REGULARITY
}

\author{
A. F. M. TER ELST and DEREK W. ROBINSON
}

(Received 26 March 1992)

Communicated by I. Raeburn

\begin{abstract}
Let $(\mathscr{X}, G, U)$ be a continuous representation of the Lie group $G$ by bounded operators $g \mapsto U(g)$ on the Banach space $\mathscr{X}$ and let $(\mathscr{X}, \mathfrak{g}, d U)$ denote the representation of the Lie algebra $\mathfrak{g}$ obtained by differentiation. If $a_{1}, \ldots, a_{d^{\prime}}$ is a Lie algebra basis of $\mathfrak{g}$ and $A_{i}=d U\left(a_{i}\right)$ then we examine elliptic regularity properties of the subelliptic operators

$$
H=-\sum_{i, j=1}^{d^{\prime}} c_{i j} A_{i} A_{j}+\sum_{i=1}^{d^{\prime}} c_{i} A_{i}+c_{0} I
$$

where $\left(c_{i j}\right)$ is a real-valued strictly positive-definite matrix and $c_{0}, c_{1}, \ldots, c_{d^{\prime}} \in \mathbb{C}$. We first introduce a family of Lipschitz subspaces $\mathscr{X}_{\gamma}, \gamma>0$, of $\mathscr{X}$, which interpolate between the $C^{n}$-subspaces of the representation and for which the parameter $\gamma$ is a continuous measure of differentiability. Secondly, we give a variety of characterizations of the spaces in terms of the semigroup generated by the closure $\bar{H}$ of $H$ and the group representation. Thirdly, for sufficiently large values of $\operatorname{Re} c_{0}$ the fractional powers of the closure of $H$ are defined, and we prove that $D\left(\bar{H}^{\gamma}\right) \subseteq \mathscr{X}_{\gamma^{\prime}}$ for $\gamma^{\prime}<2 \gamma / r$ where $r$ is the rank of the basis. Further we establish that $2 \gamma / r$ is the optimal regularity value and it is attained for unitary representations or for the representations obtained by restricting $U$ to $\mathscr{X}_{\gamma}$. Many other regularity properties are obtained.
\end{abstract}

1991 Mathematics subject classification (Amer. Math. Soc.): 43A65, 41A05, 22E45.

\section{Introduction}

The elliptic regularity theorem states that the domain of a closed second-order elliptic operator consists of functions which are once differentiable with Hölder continuous derivatives. In many cases the domain is the twice differentiable functions but this is not true in general. For example, if $\Delta$ is the usual Laplacian on $L_{p}\left(\mathbb{R}^{d} ; d x\right)$, with $d \geq 2$, then the domain $D(\Delta)$ consists of the twice differentiable functions $L_{p ; 2}$ whenever $p \in\langle 1, \infty\rangle$ but this is not the case if $p=1$ or $p=\infty$. Moreover, if

(C) 1994 Australian Mathematical Society 0263-6115/94 \$A2.00+0.00 
$p \in\langle 1, \infty\rangle$ then $D\left(\Delta^{n / 2}\right)=L_{p ; n}$, the $n$-times differentiable functions, and the graph norm on $D\left(\Delta^{n / 2}\right)$ is equivalent to the $C^{n}$-norm on $L_{p ; n}$, for each $n \in \mathbb{N}$. Thus the differential structure on $L_{p}$ is determined by the Laplacian whenever $p \in\langle 1, \infty\rangle$. These results are closely related to singular integration theory [24] and to fundamental properties of elliptic differential operators and harmonic analysis.

It is natural to examine analogous properties for continuous representations of a Lie group as a foundation for the development of the techniques of partial differential equations and harmonic analysis in the general setting. Nelson [15] obtained the first results in this direction for unitary representations. By differentiation of the unitary representation one obtains a representation of the corresponding Lie algebra by skew-self adjoint operators. Then if $A_{1}, \ldots, A_{d}$ are the infinitesimal generators corresponding to a vector space basis of the Lie algebra and $\Delta=-\sum_{i=1}^{d} A_{i}^{2}$ the corresponding Laplacian Nelson demonstrated that for each positive even integer $n$ the domain $D\left(\Delta^{n / 2}\right)$ coincides with the common domain of all monomials of order $n$ in the $A_{i}$, that is, the domain of $\Delta^{n / 2}$ is exactly the subspace spanned by the elements of the representation space which are $n$-times differentiable. Moreover, the graph norm on $D\left(\Delta^{n / 2}\right)$ is equivalent to the $C^{n}$-norm. A straightforward proof of this result and extensions to more general strongly elliptic operators without the restriction to even $n$ is described in [22, Chapter I, Section 6]. A similar situation occurs for strongly elliptic operators associated with the left, or right, regular representation on a Lie group on the $L_{p}$-spaces defined with respect to the left, or right, invariant Haar measure $([5,4])$. This also happens for particular classes of representations such as Lipschitz representations ([20,22]), or principal series representations [7]. Thus these representations, together with the unitary representations, have greater regularity properties than could be expected in general.

Our purpose is to continue this analysis of regularity properties of representations of a Lie group. But in the framework of non-commutative groups a new set of problems occur involving sublaplacians and more general subelliptic operators. Let $A_{1}, \ldots, A_{d^{\prime}}$ denote the representatives of an algebraic basis of the Lie algebra obtained by differentiation of a continuous Banach space representation of the Lie group. Then the corresponding sublaplacian is defined by $\Delta_{s}=-\sum_{i=1}^{d^{\prime}} A_{i}^{2}$ and the subelliptic regularity problem consists of comparing $D\left(\Delta_{s}^{n / 2}\right)$ with the common domain of all monomials of order $n$ in the $A_{i}$ with $1 \leq i \leq d^{\prime}$, that is, the subspace spanned by the elements of the representation space which are $n$-times differentiable in the directions of the algebraic basis. Our aim is to prove that most of the regularity results obtained previously for the Laplacian and the vector space basis extend to the sublaplacian and the algebraic basis. In particular all the results for the unitary representations have natural extensions. Nevertheless there are significant differences between the elliptic and the subelliptic theories.

Nelson's results, as derived in [22], are based on some simple features of unitary 
representations and the observation that $(\operatorname{ad} \Delta)\left(A_{i}\right)$ is a second-order polynomial in the $A_{j}$. If one tries to extend the same arguments to the subelliptic setting then problems occur because the corresponding second-order polynomial $\left(\operatorname{ad} \Delta_{s}\right)\left(A_{i}\right)$ involves $A_{j}$ with $j>d^{\prime}$, that is, the commutation relations introduce derivatives which are not in the directions of the algebraic basis. Therefore more sophisticated techniques appear essential to handle the subelliptic regularity problem.

Rothschild and Stein [23] derived regularity results for subelliptic operators acting on function spaces over a manifold $M$. In particular, if $M$ is a Lie group then their results apply to the $L_{p}$-spaces over the group, the corresponding Sobolev spaces and to various related interpolation spaces. These results were derived by combination of two techniques. The first involves embedding the group into a nilpotent group of higher dimension. The second consists of adapting the parametrix method to make estimates on the resolvents of the subelliptic operators. The disadvantage of the Rothschild-Stein results is that they are restricted to representations of the group by translations on function spaces and they are local in nature. The only global regularity results for subelliptic operators seem to be restricted to specific groups such as the Heisenberg group (see, for example, [1, Chapter I]).

Our aim is to derive global regularity results for arbitrary Banach space representations and to obtain the subelliptic analogues of Nelson's results for unitary representations and Lipschitz representations. These results are derived by quite different methods to those of Rothschild and Stein. We have no recourse to parametrix estimates and we do not use nilpotency arguments. The key ingredients of our proofs are interpolation theory and bounds on the left derivatives of the semigroup kernel associated with the sublaplacian $\Delta_{s}$. The appropriate bounds were derived for stratified groups by Jerison and Sánchez-Calle [10] and the required result for general groups follows from Varopoulos' version of the Harnack inequality $([26,27])$.

We adopt the notation of [22] throughout. In particular $(\mathscr{X}, G, U)$ denotes a strongly, or weakly*, continuous representation of the Lie group $G$ by bounded operators $g \mapsto U(g)$ on the Banach space $\mathscr{X}$. Then if $a_{i}$ is an element of the Lie algebra $\mathfrak{g}$ of $G$ we define its representative $d U\left(a_{i}\right)$ to be the infinitesimal generator of the oneparameter group $t \mapsto U\left(\exp \left(t a_{i}\right)\right)$ and we set $A_{i}=d U\left(a_{i}\right)$. Our analysis involves subelliptic operators of second-order constructed from an algebraic basis $a_{1}, \ldots, a_{d^{\prime}}$ of $\mathfrak{g}$, that is, a family which generates $\mathfrak{g}$ algebraically, as in [22, Chapter IV]. Typically the subelliptic operators have the form

$$
H=-\sum_{i, j=1}^{d^{\prime}} c_{i j} A_{i} A_{j}+\sum_{i=1}^{d^{\prime}} c_{i} A_{i}+c_{0} I,
$$

where $C=\left(c_{i j}\right)$ is a real-valued strictly positive-definite matrix and $c_{0}, c_{i} \in \mathbb{C}$. Jørgensen [11] and Robinson [22] have demonstrated that if $c_{0}, c_{i} \in \mathbb{R}$ then the closure of each such operator generates a continuous holomorphic semigroup $S$. But 
the first-order terms are a small perturbation, as a corollary of the estimates of [22, Corollary IV.4.19], and hence the closure of the operator with complex first-order terms is the generator of a holomorphic semigroup by the perturbation theory of holomorphic semigroups. As a consequence of our discussion of regularity properties we will derive a variety of properties of the action of these semigroups.

If $a_{1}, \ldots, a_{d^{\prime}}$ is an algebraic basis of $\mathfrak{g}$ let $\mathfrak{g}_{1}$ denotes its linear span and $\mathfrak{g}_{n}$ the linear span of $a_{1}, \ldots, a_{d^{\prime}}$ together with all commutators $\left(\operatorname{ad} a_{i_{1}}\right) \ldots\left(\operatorname{ad} a_{i_{k-1}}\right)\left(a_{i_{k}}\right)$ of the $a_{i}$ with $k \in\{2, \ldots, n\}$. Then $\mathfrak{g}_{1} \subset \mathfrak{g}_{2} \subset \ldots \subset \mathfrak{g}$ and the rank of $a_{1}, \ldots, a_{d^{\prime}}$ is defined to be the smallest integer $r$ such that $\mathfrak{g}_{r}=\mathfrak{g}$, for example, the rank of a vector space basis is equal to one. Note that the rank $r^{\prime}$ as defined in [22] satisfies $r^{\prime}=r-1$. Note also that if $\mathscr{X}_{n}^{\prime}, n \in \mathbb{N}$, denotes the $C^{n}$-subspace of $\mathscr{X}$ relative to $a_{1}, \ldots, a_{d^{\prime}}$ and $\mathscr{X}_{n}$ the subspace relative to the full vector space basis $a_{1}, \ldots, a_{d^{\prime}}, \ldots, a_{d}$ then

$$
\mathscr{X}_{n r}^{\prime} \subseteq \mathscr{X}_{n} \subseteq \mathscr{X}_{n}^{\prime}
$$

by the definition of $r$. Moreover, if $\mathscr{X}_{n}^{\prime}$, and $\mathscr{X}_{n}$, are equipped with the $C^{n}$-norms $\|\cdot\|_{n}^{\prime}$, and $\|\cdot\|_{n}$, respectively, as in [22], then the foregoing inclusions are continuous embeddings.

Our discussion of regularity properties is based on the theory of Lipschitz spaces which interpolate between the representation space $\mathscr{X}$ and the $C^{n}$-subspaces $\mathscr{X}_{n}^{\prime}$, or $\mathscr{X}_{n}$. The structure of these spaces was analyzed for $n=1$ and interpolation between $\mathscr{X}$ and $\mathscr{X}_{1}$ in $([20,22])$ and the general case has been considered by Pesenson $([16,17,18])$. We demonstrate that the differential structure on the Lipschitz spaces relative to the algebraic basis $a_{1}, \ldots, a_{d^{\prime}}$ is determined by each of the subelliptic operators $H$ in the same way that the differential structure on $L_{p}, p \in\langle 1, \infty\rangle$ is determined by the Laplacian. This then allows the deduction of bounds on the original space $\mathscr{X}$ of the form

$$
\|x\|_{n} \leq C\left(\left\|\bar{H}^{N} x\right\|+\|x\|\right)
$$

for all $x \in D\left(\bar{H}^{N}\right)$ where $N$ is any integer satisfying $N>n r / 2$. The $L_{p}$-examples cited above show that this is the optimal value in general although in special cases one can have bounds for $N=n r / 2$. We establish that this improvement can indeed be made for unitary representations.

\section{Interpolation spaces}

The first key ingredient in our analysis is the family of spaces which interpolate, in the sense of Peetre, between the $C^{n}$-subspaces $\mathscr{X}_{n}^{\prime}$ of the continuous representation $(\mathscr{X}, G, U)$. In this section we summarize several structural relations satisfied by these 
spaces. Although these results are straightforward to state their derivation requires a number of alternative equivalent characterizations and considerable roundabout argument which will be given in the next section. In this respect the Lie group theory is analogous to the classical theory of interpolation spaces over $\mathbb{R}^{d}$ (see, for example, [25, Chapters 1 and 2]).

Fix an algebraic basis $a_{1}, \ldots, a_{d^{\prime}}$ of the Lie algebra $g$ and let $\mathscr{X}_{n}^{\prime}$ denote the corresponding $C^{n}$-subspace of the representation space $\mathscr{X}$ and $\|\cdot\|_{n}^{\prime}$ the $C^{n}$-norm. Thus $\mathscr{X}_{0}^{\prime}=\mathscr{X},\|\cdot\|_{0}^{\prime}=\|\cdot\|$, and the higher spaces are given inductively by

$$
\mathscr{X}_{n}^{\prime}=\bigcap_{k=1}^{d^{\prime}}\left\{x \in D\left(A_{k}\right): A_{k} x \in \mathscr{X}_{n-1}^{\prime}\right\},
$$

and

$$
\|x\|_{n}^{\prime}=\sup _{k \in\left\{1, \ldots, d^{\prime}\right\}}\left\|A_{k} x\right\|_{n-1}^{\prime} .
$$

Next for $n_{1}, n_{2} \in \mathbb{N}_{0}, n_{1}<n_{2}$ and $x \in \mathscr{X}$ define the interpolation function $\kappa_{x}^{\left(n_{1}, n_{2}\right)}$ : $\langle 0, \infty\rangle \rightarrow[0, \infty]$ by setting

$$
\kappa_{x}^{\left(n_{1}, n_{2}\right)}(t)=\inf \left\{\left\|x_{n_{1}}\right\|_{n_{1}}^{\prime}+t^{n_{2}-n_{1}}\left\|x_{n_{2}}\right\|_{n_{2}}^{\prime}: x=x_{n_{1}}+x_{n_{2}}, x_{n_{1}} \in \mathscr{X}_{n_{1}}^{\prime}, x_{n_{2}} \in \mathscr{X}_{n_{2}}^{\prime}\right\}
$$

if $x \in \mathscr{X}_{n_{1}}^{\prime}$ and $\kappa_{x}^{\left(n_{1}, n_{2}\right)}(t)=\infty$ otherwise. Then for $\gamma \in\left\langle 0, n_{2}-n_{1}\right\rangle$ define $\|\cdot\|_{\gamma}^{\left(n_{1}, n_{2}\right)}: \mathscr{X} \rightarrow[0, \infty]$ by

$$
\|x\|_{\gamma}^{\left(n_{1}, n_{2}\right)}=\left(\int_{0}^{1} d t t^{-1}\left(t^{-\gamma} \kappa_{x}^{\left(n_{1}, n_{2}\right)}(t)\right)^{p}\right)^{1 / p}
$$

where $p \in[1, \infty)$ and we adopt the convention $\infty^{p}=\infty^{1 / p}=\infty$. Finally the interpolation space $\left(\mathscr{X}_{n_{1}}^{\prime}, \mathscr{X}_{n_{2}}^{\prime}\right)_{\gamma}$ is defined by

$$
\left(\mathscr{X}_{n_{1}}^{\prime}, \mathscr{X}_{n_{2}}^{\prime}\right)_{\gamma}=\left\{x \in \mathscr{X}:\|x\|_{\gamma}^{\left(n_{1}, n_{2}\right)}<\infty\right\} .
$$

It is a Banach space with respect to the norm $\|\cdot\|_{\gamma}^{\left(n_{1}, n_{2}\right)}$ and $\mathscr{X}_{n_{2}}^{\prime}$ is a norm dense subspace (see, for example, $[6, \S 3.2]$ ).

One can also define similar spaces for $p=\infty$ by replacing the norm by

$$
\|x\|_{\gamma}^{\left(n_{1}, n_{2}\right)}=\sup _{t \in\{0,1]} t^{-\gamma} \kappa_{x}^{\left(n_{1}, n_{2}\right)}(t) \quad(x \in \mathscr{X})
$$

and the principal results of the sequel are also valid in this case but there are some minor topological distinctions. We will only give complete proofs for values of $p \in[1, \infty)$ but will comment on the distinctive features of the $p=\infty$ case. The $p=\infty$ spaces are of some interest for comparison with the regularity results of Rothschild and Stein [23] mentioned in the introduction. 
The operators $A_{i}, i \in\left\{1, \ldots, d^{\prime}\right\}$ are defined, by restriction, on the interpolation spaces and the corresponding $C^{n}$-subspaces are denoted by $\left(\mathscr{X}_{n_{1}}, \mathscr{X}_{n_{2}}\right)_{\gamma ; n}$ and the $C^{n}$-norms by $\|\cdot\|_{\gamma ; n}^{\left(n_{1}, n_{2}\right)}$. Explicitly $\left(\mathscr{X}_{n_{1}}, \mathscr{X}_{n_{2}}\right)_{\gamma ; n}$ is the common domain of all $n$-th order monomials $M_{n}$ in the $A_{1}, \ldots, A_{d^{\prime}}$ and

$$
\|x\|_{\gamma ; n}^{\left(n_{1}, n_{2}\right)}=\sup \left\|M_{m} x\right\|_{\gamma}^{\left(n_{1}, n_{2}\right)}
$$

where the supremum is over all monomials of order $m \leq n$ with the convention $M_{0}=I$.

The first result is one of reduction.

THEOREM 2.1. I. If $k<n$ and $\gamma \in\langle k, n\rangle$ then $\left(\mathscr{X}, \mathscr{X}_{n}^{\prime}\right)_{\gamma}=\left(\mathscr{X}_{k}^{\prime}, \mathscr{X}_{n}^{\prime}\right)_{\gamma-k}$. II. If $\gamma \in\left\langle 0, n_{1} \wedge n_{2}\right\rangle$ then $\left(\mathscr{X}, \mathscr{X}_{n_{1}}^{\prime}\right)_{\gamma}=\left(\mathscr{X}, \mathscr{X}_{n_{2}}^{\prime}\right)_{\gamma}$.

III. If $\gamma \in\langle 0, n\rangle$ and $k \in \mathbb{N}$ then $\left(\mathscr{X}, \mathscr{X}_{n}^{\prime}\right)_{\gamma ; k}=\left(\mathscr{X}, \mathscr{X}_{n+k}^{\prime}\right)_{\gamma+k}$.

IV. If $\gamma \in\langle 0, n\rangle$ and $p \in[1, \infty)$ then $\mathscr{X}_{\infty}$ is dense in $\left(\mathscr{X}, \mathscr{X}_{n}^{\prime}\right)_{\gamma}$.

Statement I indicates that one may restrict attention to the case $k=0$. Alternatively, combination of Statements I and II shows that it suffices to examine $\gamma \in\langle 0,1]$. For example, if $\gamma \in\langle 0, n-k\rangle$ and $\bar{\gamma}$ is the smallest integer which is strictly larger than $\gamma$ and $\underline{\gamma}$ is the largest integer which is strictly smaller than $\gamma$, then

$$
\left(\mathscr{X}_{k}^{\prime}, \mathscr{X}_{n}^{\prime}\right)_{\gamma}=\left(\mathscr{X}, \mathscr{X}_{n}^{\prime}\right)_{\gamma+k}=\left(\mathscr{X}, \mathscr{X}_{\bar{\gamma}+k}^{\prime}\right)_{\gamma+k}=\left(\mathscr{X}_{\underline{\gamma}+k}^{\prime}, \mathscr{X}_{\bar{\gamma}+k}^{\prime}\right)_{\gamma-\underline{\gamma}} .
$$

Statement III indicates that $\gamma$ is to be interpreted as a fractional derivative.

Statements I, II and III have already been given by Pesenson [17] but without a complete proof. The arguments we give to establish these identities appear to differ from the ideas of Pesenson. Our proof of Theorem 2.1 is based upon several alternative characterizations of the interpolation spaces in terms of the representation $U$, or the semigroups $S$ generated by the second-order subelliptic operators, and Lipschitz conditions governed by the parameter $\gamma$.

\section{Lipschitz spaces}

The purpose of this section is threefold. First, we introduce a variety of Lipschitz spaces associated with the representation of the Lie group. Secondly, we establish identifications between these spaces and the interpolation spaces introduced in Section 2. Thirdly, we use these results to prove the reduction theorem, Theorem 2.1. Although these proofs appear extremely circuitous the line of argument follows the classical theory for function spaces over $\mathbb{R}^{d}$ (see [25, Chapters 1 and 2]) and it is not expected that there should be any direct proofs of the main structural theorems. Indeed there are considerable additional difficulties introduced in the current context 
which are not encountered in the classical theory. These difficulties arise from the non-commutative structure of the Lie group, the subellipticity restrictions and the need to cover weakly* continuous representations.

Let $\mathscr{O}$ be a bounded open neighbourhood of the identity $e$ of $G$ and $n \in \mathbb{N}$. Then for each $\gamma \in\langle 0, n\rangle$ define $\|\cdot\|_{\gamma}^{n, U}: \mathscr{X} \rightarrow[0, \infty]$ by

$$
\|x\|_{\gamma}^{n, U}=\|x\|+\left(\int_{\sigma^{n}} d \mu_{n}(g)\left(|g|^{-\gamma}\left\|\left(I-U\left(g_{1}\right)\right) \ldots\left(I-U\left(g_{n}\right)\right) x\right\|\right)^{p}\right)^{1 / p},
$$

where $g=\left(g_{1}, \ldots, g_{n}\right),|g|=\left|g_{1}\right|^{\prime}+\ldots+\left|g_{n}\right|^{\prime}$ and $|\cdot|^{\prime}$ denotes the canonical modulus function on $G$ associated with $a_{1}, \ldots, a_{d^{\prime}}$ (see [22, Section IV.4c]). Moreover, $\mu_{n}$ is the absolutely continuous measure with respect to the left invariant Haar measure on $G^{n}$ with density $g \mapsto|g|^{-n D^{\prime}}$ where $D^{\prime}$ is the local dimension corresponding to the algebraic basis $a_{1}, \ldots, a_{d^{\prime}}$. Then the Lipschitz space $\mathscr{X}_{\gamma}^{n}(U)$ is defined by

$$
\mathscr{X}_{\gamma}^{n}(U)=\left\{x \in \mathscr{X}:\|x\|_{\gamma}^{n, U}<\infty\right\} .
$$

It is a Banach space with respect to the norm $\|\cdot\|_{\gamma}^{n, U}$. Note that as $p$ is fixed throughout we have suppressed it in the notation. Moreover, since the space is independent of the choice of $\mathscr{O}$, up to equivalence of norms, we have also omitted it from the notation.

Next we introduce a uniform version of the Lipschitz spaces. First, for each $x \in \mathscr{X}$ and $n \in \mathbb{N}_{0}$ define $\omega_{x}^{(n)}:\langle 0, \infty\rangle \rightarrow[0, \infty\rangle$ by $\omega_{x}^{(0)}(t)=\|x\|$ and

$$
\omega_{x}^{(n)}(t)=\sup _{\substack{g_{1}, \ldots, g_{n} \in G \\\left|g_{j}\right|^{\prime} \leq t}}\left\|\left(I-U\left(g_{1}\right)\right) \ldots\left(I-U\left(g_{n}\right)\right) x\right\|
$$

for $n \in \mathbb{N}$. Secondly, for $\gamma \in\langle 0, n\rangle$ define $\|\cdot\|_{\gamma}^{n, \omega}: \mathscr{X} \rightarrow[0, \infty]$ by

$$
\|x\|_{\gamma}^{n, \omega}=\|x\|+\left(\int_{0}^{1} d t t^{-1}\left(t^{-\gamma} \omega_{x}^{(n)}(t)\right)^{p}\right)^{1 / p} .
$$

Then the space

$$
\mathscr{X}_{\gamma}^{n, \omega}=\left\{x \in \mathscr{X}:\|x\|_{\gamma}^{n, \omega}<\infty\right\}
$$

is a Banach space with respect to the norm $\|\cdot\|_{\gamma}^{n, \omega}$.

Finally, if $S$ is a continuous semigroup on $\mathscr{X}$ we introduce the corresponding Lipschitz spaces as follows. For $n \in N$ and $\gamma \in\langle 0, n\rangle$ define $\|\cdot\|_{\gamma}^{n, S}: \mathscr{X} \rightarrow[0, \infty]$ by

$$
\|x\|_{\gamma}^{n, S}=\|x\|+\left(\int_{0}^{1} d t t^{-1}\left(t^{-\gamma}\left\|\left(I-S_{t}\right)^{n} x\right\|\right)^{p}\right)^{1 / p} .
$$

Then

$$
\mathscr{X}_{\gamma}^{n, S}=\left\{x \in \mathscr{X}:\|x\|_{\gamma}^{n, S}<\infty\right\}
$$


is a Banach space with respect to the norm $\|\cdot\|_{\gamma}^{n, S}$.

It is useful to note that if $\rho \in \mathbb{R}$ and $T$ is the semigroup defined by $T_{t}=S_{t} e^{-\rho t}$, $t>0$, then $\mathscr{X}_{\gamma}^{n, S}=\mathscr{X}_{\gamma}^{n, T}$ and the norms are equivalent. This follows from the equality

$$
I-T_{t}=\left(I-S_{t}\right) e^{-\rho t}+\left(I-e^{-\rho t}\right) I
$$

and the inequalities

$$
\left\|\left(\left(I-S_{t}\right) / t\right)^{k} x\right\| \leq \varepsilon^{n-k}\left\|\left(\left(I-S_{t}\right) / t\right)^{n} x\right\|+c \varepsilon^{-k}\|x\|
$$

for some $c>0$, uniform in $t \in\langle 0,1], \varepsilon \in\langle 0,1]$ and $x \in \mathscr{X}$.

Furthermore, if $S$ is equibounded, define $\|\cdot\|_{\gamma}^{n, S, \infty}: \mathscr{X} \rightarrow[0, \infty]$ by

$$
\|x\|_{\gamma}^{n . S . \infty}=\|x\|+\left(\int_{0}^{\infty} d t t^{-1}\left(t^{-\gamma}\left\|\left(I-S_{t}\right)^{n} x\right\|\right)^{p}\right)^{1 / p} .
$$

Then

$$
\mathscr{X}_{\gamma}^{n, S, \infty}=\left\{x \in \mathscr{X}:\|x\|_{\gamma}^{n, S, \infty}<\infty\right\}
$$

is a Banach space with respect to the norm $\|\cdot\|_{\gamma}^{n, S, \infty}$. Moreover, $\mathscr{X}_{\gamma}^{n, S, \infty}=\mathscr{X}_{\gamma}^{n, S}$, with equivalent norms (see [6, Section 3.4.1]).

If $S$ is a holomorphic exponentially decreasing semigroup, there exists another description of $\mathscr{X}_{\gamma}^{n, S, \infty}$, due to Peetre. Let $H$ be the generator of $S$. For $\gamma \in\langle 0, n\rangle$ define $\|\cdot\|_{\gamma}^{n, H}: \mathscr{X} \rightarrow[0, \infty]$ by

$$
\|x\|_{\gamma}^{n, H}=\|x\|+\left(\int_{0}^{\infty} d t t^{-1}\left(t^{n-\gamma}\left\|H^{n} S_{t} x\right\|\right)^{p}\right)^{1 / p} .
$$

Then the space

$$
\mathscr{X}_{\gamma}^{n, H}=\left\{x \in \mathscr{X}:\|x\|_{\gamma}^{n, H}<\infty\right\}
$$

is a Banach space with respect to the norm $\|\cdot\|_{\gamma}^{n, H}$.

Finally we note that all the above spaces have $p=\infty$ analogues. The $L_{p}$-norms are replaced by the supremum norm.

For every interpolation pair $(\mathscr{Y}, \mathscr{Z})$ and $\gamma \in\langle 0,1\rangle$ let $(\mathscr{Y}, \mathscr{Z})_{\gamma, p: \mathrm{K}}$ be the interpolation space by the K-method of Peetre (see [6, Definition 3.2.4]). For any operator $T$ on $\mathscr{X}$ equip the domain $D(T)$ with the graph norm $x \mapsto\|T x\|+\|x\|$. Note that $D(T)$ is a Banach space if, and only if, $T$ is a norm closed operator. Moreover, note that every weakly* closed operator is norm closed.

THEOREM 3.1. Let $n>\gamma>0$. Let $S$ be a holomorphic exponentially decreasing semigroup with generator $H$. Then

$$
\mathscr{X}_{\gamma}^{n, S, \infty}=\mathscr{X}_{\gamma}^{n, H}=\left(\mathscr{X}, D\left(H^{n}\right)\right)_{\gamma / n, p ; \mathrm{K}}
$$


and the norms are equivalent. Moreover, if $n^{\prime} \in \mathbb{N}$ and $n^{\prime}>\gamma$ then $\mathscr{X}_{\gamma}^{n^{\prime}, S, \infty}=\mathscr{X}_{\gamma}^{n, S, \infty}$ and the norms are equivalent.

If the semigroup is weakly* continuous let $\widetilde{\mathscr{X}}$ be the subspace of $\mathscr{X}$ on which $S$ acts strongly continuously and let $\widetilde{S}$ be the restriction of $S$ to $\widetilde{\mathscr{X}}$. Then

$$
\mathscr{X}_{\gamma}^{n, S, \infty}=\widetilde{\mathscr{X}}_{\gamma}^{n, \tilde{s}, \infty}
$$

PROOF. First suppose the semigroup is strongly continuous. The first two equalities are proved in [6, Section 3.5 and Theorem 3.4.2]. The third one follows from [6, Theorem 3.4.6].

Secondly, suppose the semigroup is weakly* continuous. Let $\tilde{H}$ be the infinitesimal generator of the strongly continuous semigroup $\widetilde{S}$. Clearly $\|x\|_{\gamma}^{n, S, \infty}=$ $\|x\|_{\gamma}^{n, \widetilde{S}, \infty}$ for all $x \in \widetilde{\mathscr{X}}$ and similarly for the norms on $\mathscr{X}_{\gamma}^{n, H}$ and $\widetilde{\mathscr{X}}_{\gamma}^{n, \widetilde{H}}$. Moreover, $\left(\mathscr{X}, D\left(H^{n}\right)\right)_{\gamma / n, p ; \mathrm{K}}$ and $\left(\widetilde{\mathscr{X}}, D\left(\tilde{H}^{n}\right)\right)_{\gamma / n, p ; \mathrm{K}}$ coincide on $\frac{\mathcal{X}}{\mathscr{X}}$. So it remains to prove that $\mathscr{X}_{\gamma}^{n, S, \infty}, \mathscr{X}_{\gamma}^{n, H}$ and $\left(\mathscr{X}, D\left(H^{n}\right)\right)_{\gamma / n, p ; \mathrm{K}}$ are subspaces of $\widetilde{\mathscr{X}}$.

Let $\mu \in\langle 0,1 \wedge \gamma\rangle$. We will show that

$$
\mathscr{X}_{\gamma}^{n, H} \subseteq \mathscr{X}_{\mu}^{1, H} \subseteq \widetilde{X}
$$

Using the identity

$$
S_{t} x=\int_{t}^{\infty} d s s^{-1} H S_{s} x
$$

and the Hardy inequality, [6, Lemma 3.4.7], we obtain by induction that

$$
\begin{aligned}
& \left(\int_{0}^{\infty} d t t^{-1}\left(t^{1-\mu}\left\|H S_{t} x\right\|\right)^{p}\right)^{1 / p} \\
& \quad \leq(1-\mu)^{-1} \ldots(n-1-\mu)^{-1}\left(\int_{0}^{\infty} d t t^{-1}\left(t^{n-\mu}\left\|H^{n} S_{t} x\right\|\right)^{p}\right)^{1 / p}
\end{aligned}
$$

for all $x \in \mathscr{X}$. Let $M, \rho>0$ be such that $\left\|S_{t}\right\| \leq M e^{-\rho t}$ for all $t>0$. Then $\left\|H^{n} S_{t}\right\| \leq M\left\|H^{n} S_{1}\right\| e^{-\rho(t-1)}$. So there exists a $c>0$ such that

$$
\left(\int_{0}^{\infty} d t t^{-1}\left(t^{1-\mu}\left\|H S_{t} x\right\|\right)^{p}\right)^{1 / p} \leq c\|x\|+\left(\int_{0}^{1} d t t^{-1}\left(t^{n-\mu}\left\|H^{n} S_{t} x\right\|\right)^{p}\right)^{1 / p}
$$

for all $x \in \mathscr{X}$. Hence

$$
\begin{aligned}
\|x\|_{\mu}^{1, H} & \leq(c+1)\|x\|+\left(\int_{0}^{1} d t t^{-1}\left(t^{n-\mu}\left\|H^{n} S_{t} x\right\|\right)^{p}\right)^{1 / p} \\
& \leq(c+1)\|x\|+\left(\int_{0}^{1} d t t^{-1}\left(t^{n-\lambda}\left\|H^{n} S_{t} x\right\|\right)^{p}\right)^{1 / p} \leq(c+1)\|x\|_{\lambda}^{n, H}
\end{aligned}
$$


for all $x \in \mathscr{X}$. So $\mathscr{X}_{\gamma}^{n, H} \subseteq \mathscr{X}_{\mu}^{1, H}$.

Now let $x \in \mathscr{X}_{\mu}^{1, H}$. Then for all $t \in\langle 0,1]$ one finds

$$
\begin{aligned}
\left\|\left(I-S_{t}\right) x\right\| & \leq \int_{0}^{t} d s\left\|H S_{s} x\right\| \\
& \leq\left(\int_{0}^{t} d s s^{-1+\mu q}\right)^{1 / q}\left(\int_{0}^{t} d s s^{-1}\left(s^{1-\mu}\left\|H S_{s}\right\|\right)^{p}\right)^{1 / p} \\
& \leq c t^{\mu}\|x\|_{\mu}^{1, H}
\end{aligned}
$$

for some constant $c>0$, independent of $t$ by the Hölder inequality, where $q \in[1, \infty]$ is such that $1 / p+1 / q=1$. Consequently $x \in \widetilde{\mathscr{X}}$ and hence $\mathscr{X}_{\mu}^{1, H} \subseteq \widetilde{\mathscr{X}}$.

Next, the proof of the inclusion

$$
\left(\mathscr{X}, D\left(H^{n}\right)\right)_{\gamma, p ; \mathrm{K}} \subseteq \mathscr{X}_{\gamma}^{n, H}
$$

in [6, Theorem 3.5.3] works equally well for weakly* continuous semigroups. Therefore $\left(\mathscr{X}, D\left(H^{n}\right)\right)_{\gamma, p ; \mathrm{K}} \subseteq \widetilde{\mathscr{X}}$.

Similarly, the proof of the inclusion

$$
\mathscr{X}_{\gamma}^{1, S, \infty} \subseteq \mathscr{X}_{\gamma}^{1, H}
$$

in the proof of [6, Theorem 3.5.3] does not use the strong continuity of the semigroup. It is easy to prove that

$$
\mathscr{X}_{\gamma}^{n, S, \infty}=\mathscr{X}_{\gamma}^{n, S} \subseteq \mathscr{X}_{\gamma^{\prime}}^{n, S}
$$

for all $\gamma^{\prime} \leq \gamma$. So we may restrict ourselves to the case $\gamma<1$ and $n \geq 2$. We shall prove that there exists a $\mu \in\langle 0,1\rangle$ such that

$$
\mathscr{X}_{\gamma}^{n, S} \subseteq \mathscr{X}_{\mu}^{1, S}
$$

Let $\delta=2 \gamma /(2 n-1)$ and $\mu=\delta / 2$. By setting $\varepsilon=t^{1-\delta}$ in (1) one obtains bounds

$$
\left\|\left(I-S_{t}\right) x\right\| \leq t^{-(n-1) \delta}\left\|\left(I-S_{t}\right)^{n} x\right\|+c t^{\delta}\|x\|
$$

uniform in $t \in\langle 0,1]$ and $x \in \mathscr{X}$. So

$$
\begin{aligned}
& \left(\int_{0}^{1} d t t^{-1}\left(t^{-\mu}\left\|\left(I-S_{t}\right) x\right\|\right)^{p}\right)^{1 / p} \\
& \quad \leq\left(\int_{0}^{1} d t t^{-1}\left(t^{-\mu-(n-1) \delta}\left\|\left(I-S_{t}\right)^{n} x\right\|\right)^{p}\right)^{1 / p}+c\left(\int_{0}^{1} d t t^{-1}\left(t^{\delta-\mu}\|x\|\right)^{p}\right)^{1 / p} \\
& \quad \leq\|x\|_{\gamma}^{n, S}+c((\delta-\mu) p)^{-1 / p}\|x\| .
\end{aligned}
$$

This proves the theorem. 
Now the key characterizations of the interpolation spaces are provided by the following theorem.

THEOREM 3.2. For each $n \in \mathbb{N}$ and each $\gamma \in\langle 0, n\rangle$

$$
\left(\mathscr{X}, \mathscr{X}_{n}^{\prime}\right)_{\gamma}=\mathscr{X}_{\gamma}^{n, \omega}=\mathscr{X}_{\gamma}^{n}(U)=\mathscr{X}_{\gamma / 2}^{n, S}
$$

and the norms $\|\cdot\|_{\gamma}^{(0, n)},\|\cdot\|_{\gamma}^{n, \omega},\|\cdot\|_{\gamma}^{n, U}$ and $\|\cdot\|_{\gamma / 2}^{n, S}$ are equivalent, where $S$ denotes the continuous holomorphic semigroup generated by the closure of the sublaplacian

$$
H=-\sum_{i=1}^{d^{\prime}} A_{i}^{2}
$$

REMARK. The sublaplacian seems to play a distinguished role in the characterization of the interpolation spaces but this is illusory. The theorem will be used in Section 5 to deduce that if $S$ denotes the semigroup generated by the secondorder subelliptic operator $H$ described in the introduction then the interpolation space $\left(\mathscr{X}, \mathscr{X}_{n}^{\prime}\right)_{\gamma}$ can be identified with the space $\mathscr{X}_{\gamma / 2}^{n, S}$.

PROOF. The proof is in four steps. Each step establishes one of the inclusions

$$
\left(\mathscr{X}, \mathscr{X}_{n}^{\prime}\right)_{\gamma} \subseteq \mathscr{X}_{\gamma}^{n, \omega} \subseteq \mathscr{X}_{\gamma}^{n}(U) \subseteq \mathscr{X}_{\gamma / 2}^{n, S} \subseteq\left(\mathscr{X}, \mathscr{X}_{n}^{\prime}\right)_{\gamma}
$$

and simultaneously establishes continuity of the inclusion. The continuity also follows easily from the closed graph theorem.

Step $1 \quad\left(\mathscr{X}, \mathscr{X}_{n}^{\prime}\right)_{\gamma} \subseteq \mathscr{X}_{\gamma}^{n, \omega}$. If $x \in \mathscr{X}$ and $x=x_{0}+x_{n}$ with $x_{0} \in \mathscr{X}$ and $x_{n} \in \mathscr{X}_{n}^{\prime}$ then

$$
\omega_{x}^{(n)}(t) \leq \omega_{x_{0}}^{(n)}(t)+\omega_{x_{n}}^{(n)}(t) .
$$

for all $t \in\langle 0,1]$. Since $B_{r}^{\prime}=\left\{g \in G:|g|^{\prime} \leq r\right\}$ is compact for each $r>0$ there exist an $M \geq 1$ such that $\|U(g)\| \leq M$ for all $g \in B_{4}^{\prime}$. Therefore $\omega_{x_{0}}^{(n)}(t) \leq(1+M)^{n}\left\|x_{0}\right\|$ for all $t \in\langle 0,1]$.

Next, following an idea of Pesenson [18], we prove that

$$
\omega_{x_{1}}^{(l)}(t) \leq(M+2) d^{\prime} t \sup _{k \in\left\{1, \ldots, d^{\prime}\right\}} \omega_{A_{k} x_{1}}^{(l-1)}((1+\varepsilon) t)
$$

for all $l \in \mathbb{N}$, all $x_{1} \in \mathscr{X}_{1}^{\prime}$, all $t \in\langle 0,2]$ and all $\varepsilon \in\langle 0,1]$, where $M$ is as above. For $a_{i} \in \mathfrak{g}$ let $\widetilde{A_{i}}$ be the corresponding left invariant vector field on $G$. So

$$
\left(\widetilde{A_{i}} \psi\right)(g)=\left.\frac{d}{d t} \psi\left(g \exp \left(t a_{i}\right)\right)\right|_{t=0}
$$


for every $C^{\infty}$-function $\psi$ on $G$. In particular if

$$
\psi(h)=\left(U(h) x_{1}, f\right)
$$

where $x_{1} \in \mathscr{X}_{1}^{\prime}$ and $f \in \mathscr{F}_{\infty}$, the space of $C^{\infty}$-elements for the adjoint representation on the dual, or predual, $\mathscr{F}$, then

$$
\left(\widetilde{A}_{i} \psi\right)(h)=\left(U(h) A_{i} x_{1}, f\right) .
$$

Now if $\varphi:[0,1] \rightarrow G$ is an absolutely continuous path from $e$ to $g_{l}$ with tangential co-ordinates $\varphi_{i}$ in the directions $\widetilde{A}_{i}$ one has

$$
\begin{aligned}
\left(\left(I-U\left(g_{l}\right)\right) x_{1}, f\right) & =\psi(e)-\psi\left(g_{l}\right) \\
& =-\int_{0}^{1} d s \sum_{i=1}^{d^{\prime}} \varphi_{i}(s)\left(U(\varphi(s)) A_{i} x_{1}, f\right) \\
& =-\int_{0}^{1} d s \sum_{i=1}^{d^{\prime}} \varphi_{i}(s)\left(\left((I-U(\varphi(s))) A_{i} x_{1}, f\right)+\left(A_{i} x_{1}, f\right)\right) .
\end{aligned}
$$

But one may choose $\varphi$ such that

$$
\int_{0}^{1} d s\left(\sum_{i=1}^{d^{\prime}} \varphi_{i}(s)^{2}\right)^{1 / 2} \leq(1+\varepsilon)\left|g_{l}\right|^{\prime} .
$$

Then $|\varphi(s)|^{\prime} \leq(1+\varepsilon)\left|g_{l}\right|^{\prime}$ for all $s \in[0,1]$. Hence

$$
\begin{gathered}
\left|\left(\left(I-U\left(g_{l}\right)\right) x_{1}, f\right)\right| \leq d^{\prime}(1+\varepsilon)\left|g_{l}\right|^{\prime} \sup \left\{\left|\left(I-U(\varphi(s)) A_{i} x_{1}, f\right)\right|+\left|\left(A_{i} x_{1}, f\right)\right|\right. \\
\left.: i \in\left\{1, \ldots, d^{\prime}\right\}, s \in[0,1]\right\} .
\end{gathered}
$$

Now replacing $f$ by $\left(\left(I-U\left(g_{1}\right)\right) \ldots\left(I-U\left(g_{l-1}\right)\right)\right)^{*} f$ and taking the supremum over $f$ with $\|f\| \leq 1$ and $\left|g_{i}\right|^{\prime} \leq t$ one finds

$$
\begin{aligned}
\omega_{x_{1}}^{(l)}(t) & \leq d^{\prime}(1+\varepsilon) t \sup _{i \in\left\{1, \ldots, d^{\prime}\right\}}\left\{\omega_{A_{i} x_{1}}^{(l)}((1+\varepsilon) t)+\omega_{A_{i} x_{1}}^{(l-1)}(t)\right\} \\
& \leq d^{\prime}(1+\varepsilon)(M+2) t \sup _{i \in\left\{1, \ldots, d^{\prime}\right\}} \omega_{A_{i} x_{1}}^{(l-1)}((1+\varepsilon) t) .
\end{aligned}
$$

It now follows by iteration of this inequality that

$$
\omega_{x_{n}}^{(n)}(t) \leq\left(d^{\prime}(M+2)\right)^{n} t^{n}\left\|x_{n}\right\|_{n}^{\prime}
$$

for all $t \in\langle 0,1]$. Consequently

$$
\omega_{x}^{(n)}(t) \leq\left(d^{\prime}(M+2)\right)^{n}\left(\left\|x_{0}\right\|+t^{n}\left\|x_{n}\right\|_{n}^{\prime}\right)
$$


and by taking the infimum over all decompositions $x=x_{0}+x_{n}$ with $x_{n} \in \mathscr{X}_{n}^{\prime}$ one concludes that

$$
\omega_{x}^{(n)}(t) \leq\left(d^{\prime}(M+2)\right)^{n} \kappa_{x}^{(0, n)}(t)
$$

for all $t \in\langle 0,1]$. Therefore

$$
\|x\|_{\gamma}^{n, \omega} \leq\left(1+\left(d^{\prime}(M+2)\right)^{n}\right)\|x\|_{\gamma}^{(0, n)}
$$

and $\left(\mathscr{X}, \mathscr{X}_{n}^{\prime}\right)_{\gamma}$ is continuously embedded in $\mathscr{X}_{\gamma}^{n, \omega}$.

Step $2 \mathscr{X}_{\gamma}^{n, \omega} \subseteq \mathscr{X}_{\gamma}^{(n)}(U)$. The local dimension $D^{\prime}$ is defined such that there exists a $c \geq 1$ with the property

$$
c^{-1} r^{D^{\prime}} \leq\left|B_{r}^{\prime}\right| \leq c r^{D^{\prime}}
$$

for all $r \in\langle 0,1]$.

Choosing $\mathscr{O}=B_{1 /(2 n)}^{\prime}$ it then follows that for $x \in \mathscr{X}$

$$
\begin{aligned}
\int_{\sigma^{n}} d \mu_{n}(g)\left(|g|^{-\gamma}\left\|\left(I-U\left(g_{1}\right)\right) \ldots\left(I-U\left(g_{n}\right)\right) x\right\|\right)^{p} & \\
\leq & \int_{\sigma^{n}} d \mu_{n}(g)\left(|g|^{-\gamma} \omega_{x}^{(n)}\left(\max \left(\left|g_{1}\right|^{\prime}, \ldots,\left|g_{n}\right|^{\prime}\right)\right)\right)^{p} .
\end{aligned}
$$

Therefore by splitting the integral into a sum of integrals over the annuli

$$
\left\{g: 2^{-(l+1)}<|g| \leq 2^{-l}\right\} \cap \mathscr{O}^{n}
$$

one has

$$
\begin{aligned}
\left(\int _ { \sigma ^ { n } } d \mu _ { n } ( g ) \left(|g|^{-\gamma}||(I-\right.\right. & \left.\left.\left.U\left(g_{1}\right)\right) \ldots\left(I-U\left(g_{n}\right)\right) x \|\right)^{p}\right)^{1 / p} \\
& \leq\left(\sum_{l=1}^{\infty}\left(\left(2^{-(l+1)}\right)^{-\gamma} \omega_{x}^{(n)}\left(2^{-l}\right)\right)^{p} 2^{(l+1) n D^{\prime}}\left(c 2^{-l D^{\prime}}\right)^{n}\right)^{1 / p} \\
& =c^{n / p} 2^{\left(n D^{\prime}+1+2 p \gamma\right) / p}\left(\sum_{l=0}^{\infty}\left(\left(2^{-l}\right)^{-\gamma} \omega_{x}^{(n)}\left(2^{-(l+1)}\right)\right)^{p} 2^{-1}\right)^{1 / p} \\
& \leq c^{n / p} 2^{\left(n D^{\prime}+1+2 p \gamma\right) / p}\left(\int_{0}^{1} d t t^{-1}\left(t^{-\gamma} \omega_{x}^{(n)}(t)\right)^{p}\right)^{1 / p} .
\end{aligned}
$$

Hence $\mathscr{X}_{\gamma}^{n, \omega}$ is continuously embedded in $\mathscr{X}_{\gamma}^{(n)}(U)$.

Step $3 \mathscr{X}_{\gamma}^{(n)}(U) \subseteq \mathscr{X}_{\gamma / 2}^{n, S}$. The semigroup $S$ has a kernel $K$ with

$$
\int_{G} d g K_{t}(g)=1
$$


for all $t>0$. Therefore, for $x \in \mathscr{X}$

$$
\left(I-S_{t}\right)^{n} x=\int_{G^{n}} d g_{1} \ldots d g_{n} K_{t}\left(g_{1}\right) \ldots K_{t}\left(g_{n}\right)\left(I-U\left(g_{1}\right)\right) \ldots\left(I-U\left(g_{n}\right)\right) x
$$

and hence

$$
\begin{aligned}
& t^{-\gamma / 2}\left\|\left(I-S_{t}\right)^{n} x\right\| \\
& \quad \leq t^{-\gamma / 2} \int_{G^{n}} d g_{1} \ldots d g_{n}\left|K_{t}\left(g_{1}\right) \ldots K_{t}\left(g_{n}\right)\right|\left\|\left(I-U\left(g_{1}\right)\right) \ldots\left(I-U\left(g_{n}\right)\right) x\right\| .
\end{aligned}
$$

Now we split the multiple integral into two parts. The first which we denote by $I_{\gamma}(t)$ is the integral over $B_{r}^{\prime} \times \ldots \times B_{r}^{\prime}$ for some fixed $r \in\langle 0,1]$ and the second which we denote by $E_{\gamma}(t)$ is the remainder. Thus $E_{\gamma}(t)$ is less than or equal to the sum of $n$ terms each of which has $n-1$ integrals over $G$ and the $n$-th integral is over $G \backslash B_{r}^{\prime}$. Noting, however, that one has bounds $\|U(g)\| \leq M e^{\rho|g|^{\prime}}$ for all $g \in G$ and suitable $M \geq 1, \rho \geq 0$ one finds that

$$
E_{\gamma}(t) \leq n(2 M)^{n} t^{-\gamma / 2}\|x\|\left(\int_{G} d g\left|K_{t}(g)\right| e^{\rho|g|^{\prime}}\right)^{n-1} \int_{G \backslash B_{r}^{\prime}} d g\left|K_{t}(g)\right| e^{\rho|g|^{\prime}} .
$$

But it follows from the estimates of [22, Section IV.4], by an argument similar to that used for the derivation of [22, inequality (IV.4.32)], that one has bounds

$$
\int_{G} d g\left|K_{t}(g)\right| e^{\rho|g|^{\prime}} \leq a e^{\omega\left(1+\rho^{2}\right)}
$$

for all $t \in\langle 0,1]$ and all $\rho \geq 0$. Moreover, one has Gaussian bounds

$$
\left|K_{t}(g)\right| \leq a t^{-D^{\prime} / 2} e^{-b\left(|g|^{\prime}\right)^{2} / t}
$$

for all $g \in G$ and $t \in\langle 0,1]$ and suitable $a, b>0$ by [22, Theorem IV.4.16], and the discussion, [22, page 331]. Also there exists an $M \geq 1$ and $\omega \geq 0$ such that $\left|B_{r}^{\prime}\right| \leq M e^{\omega r}$ for all $r>0$. So by splitting the integral into a sum of integrals over the sets $\left\{N r<|g|^{\prime} \leq(N+1) r\right\}$ one finds

$$
\begin{aligned}
t^{-\gamma / 2} \int_{G \backslash B_{r}^{\prime}} d g\left|K_{t}(g)\right| e^{\rho|g|^{\prime}} & \leq a M \sum_{N=1}^{\infty} t^{-\gamma / 2} e^{-b N^{2} r^{2} / t} e^{(N+1) \rho r} e^{(N+1) \omega r} \\
& \leq a_{m} t^{m-\gamma / 2}
\end{aligned}
$$

for all $m \in \mathbb{N}$ and $t \in\langle 0,1]$. Therefore by choosing $m$ large enough one obtains estimates

$$
E_{\gamma}(t) \leq c_{l} t^{\prime}\|x\|
$$


valid for all $t \in\langle 0,1]$ and each $l>1$. Hence

$$
\left(\int_{0}^{1} d t t^{-1}\left(E_{\gamma}(t)\right)^{p}\right)^{1 / p} \leq c_{l}(l p)^{-1 / p}\|x\| .
$$

Next we consider the integral $I_{\gamma}(t)$. The function $I_{\gamma}$ can be expressed in the form $L_{\gamma} f_{Y}$ where $f_{\gamma}$ is the function over $G^{n}$ defined by

$$
f_{\gamma}(g)=|g|^{-\gamma}\left\|\left(I-U\left(g_{1}\right)\right) \ldots\left(I-U\left(g_{n}\right)\right) x\right\|
$$

and for every measurable function $f:\left(B_{r}^{\prime}\right)^{n} \rightarrow[0, \infty\rangle$ we define $L_{\gamma} f:\langle 0,1] \rightarrow$ $[0, \infty]$ by

$$
\left(L_{\gamma} f\right)(t)=t^{-\gamma / 2} \int_{\left(B_{r}^{\prime}\right)^{n}} d g_{1} \ldots d g_{n}\left|K_{t}\left(g_{1}\right) \ldots K_{t}\left(g_{n}\right)\right||g|^{\gamma} f(g) .
$$

We shall prove that there exists a $c>0$ such that

$$
\left\|L_{\gamma} f\right\|_{L_{q}(\langle 0,1] ; d t / t)} \leq c\|f\|_{L_{q}\left(\left(B_{r}^{\prime}\right)^{n} ; d \mu_{n}\right)}
$$

for all measurable $f:\left(B_{r}^{\prime}\right)^{n} \rightarrow[0, \infty\rangle$ and $q \in\{1, \infty\}$. Then one wants to define the $\operatorname{map} M_{\gamma}: L_{q}\left(\left(B_{r}^{\prime}\right)^{n} ; d \mu_{n}\right) \rightarrow L_{q}(\langle 0,1] ; d t / t)$ by

$$
\left(M_{\gamma} f\right)(t)=t^{-\gamma / 2} \int_{\left(B_{r}^{\prime}\right)^{n}} d g_{1} \ldots d g_{n}\left|K_{t}\left(g_{1}\right) \ldots K_{t}\left(g_{n}\right)\right||g|^{\gamma} f(g)
$$

and by (7) we know (8) makes sense if $q \in\{1, \infty\}$ and $M_{\gamma}$ is bounded if $q \in\{1, \infty\}$. Hence by the Riesz-Thorin interpolation theorem, [28, Theorem XII.1.11], (8) makes sense if $q \in[1, \infty]$ and $M_{\gamma}$ is also bounded if $q=p$. We prove inequality (7) for $q \in\{1, \infty\}$.

Suppose $q=1$. Then

$$
\int_{0}^{1} d t t^{-1}\left|\left(L_{\gamma} f\right)(t)\right| \leq\|f\|_{1} \sup _{g_{1}, \ldots, g_{n} \in G} \int_{0}^{1} d t t^{-1-\gamma / 2}\left|K_{t}\left(g_{1}\right) \ldots K_{t}\left(g_{n}\right)\right||g|^{\gamma+n D^{\prime}} .
$$

Now there exists a $k \in\{1, \ldots, n\}$ such that $\left|g_{k}\right|^{\prime} \geq|g| / n$. Then since $K$ satisfies Gaussian bounds

$$
\left|K_{t}(g)\right| \leq a t^{-D^{\prime} / 2} e^{-b\left(|g|^{\prime}\right)^{2} / t}
$$

for all $t \in\langle 0,1]$ and $g \in G$ it follows that

$$
\begin{aligned}
\int_{0}^{1} d t t^{-1-\gamma / 2}\left|K_{t}\left(g_{1}\right) \ldots K_{t}\left(g_{n}\right)\right||g|^{\gamma+n D^{\prime}} & \leq a^{n} n^{\gamma+n D^{\prime}} \int_{(|g| / n)^{2}}^{\infty} d x x^{-1} x^{\left(\gamma+n D^{\prime}\right) / 2} e^{-b x} \\
& \leq a^{n} n^{\gamma+n D^{\prime}} \int_{0}^{\infty} d x x^{-1} x^{\left(\gamma+n D^{\prime}\right) / 2} e^{-b x}<\infty .
\end{aligned}
$$


Next consider the case $q=\infty$. Then

$$
\begin{aligned}
\sup _{t \in\{0,1]}\left|\left(L_{\gamma} f\right)(t)\right| & \leq\|f\|_{\infty} \sup _{t \in\langle 0,1]} t^{-\gamma / 2} \int_{\left(B_{r}^{\prime}\right)^{n}} d g_{1} \ldots d g_{n}\left|K_{t}\left(g_{1}\right) \ldots K_{t}\left(g_{n}\right)\right||g|^{\gamma} \\
& \leq\|f\|_{\infty} n^{1+\gamma} \sup _{t \in\{0,1]}\left(\int_{B_{r}^{\prime}} d g\left|K_{t}(g)\right|\right)^{n-1} t^{-\gamma / 2} \int_{B_{r}^{\prime}} d g\left|K_{t}(g)\right|\left(|g|^{\prime}\right)^{\gamma} \\
& \leq\|f\|_{\infty} n^{1+\gamma}\left(a e^{\omega}\right)^{n-1} \sup _{t \in\{0,1]} t^{-\gamma / 2} \int_{B_{r}^{\prime}} d g\left|K_{t}(g)\right|\left(|g|^{\prime}\right)^{\gamma}
\end{aligned}
$$

by inequality (5). But by splitting the integral into a sum of integrals over the sets

$$
\left\{g \in G: N \leq|g|^{\prime} / t^{1 / 2}<N+1\right\} \cap B_{r}^{\prime}
$$

and using the Gaussian bounds on $K$ and the estimates (4) on the volume of the balls one obtains

$$
t^{-\gamma / 2} \int_{B_{r}^{\prime}} d g\left|K_{t}(g)\right|\left(|g|^{\prime}\right)^{\gamma} \leq a c t^{-\left(\gamma+D^{\prime}\right) / 2} \sum_{N=0}^{\infty} e^{-b N^{2}}\left((N+1) t^{1 / 2}\right)^{\gamma+D^{\prime}}
$$

which is uniformly bounded for $t \in\langle 0,1]$. So $\left\|L_{\gamma} f\right\|_{\infty} \leq C\|f\|_{\infty}$ for some $C>0$ for all positive measurable $f$.

Step $4 \quad \mathscr{X}_{\gamma / 2}^{n, S} \subseteq\left(\mathscr{X}, \mathscr{X}_{n}^{\prime}\right)_{\gamma} . \quad$ For each $x \in \mathscr{X}$

$$
\left(I-\left(I-S_{t}\right)^{n}\right) x=-\sum_{k=1}^{n}(-1)^{k}\left(\begin{array}{l}
n \\
k
\end{array}\right) S_{k t} x \in \mathscr{X}_{n}^{\prime}
$$

Therefore

$$
\kappa_{x}^{(0, n)}(t) \leq\left\|\left(I-S_{t^{2}}\right)^{n} x\right\|+t^{n} \sum_{k=1}^{n}\left(\begin{array}{l}
n \\
k
\end{array}\right)\left\|S_{k t^{2}} x\right\|_{n}^{\prime} .
$$

Consequently

$$
\|x\|_{\gamma}^{(0, n)} \leq 2^{-1 / p}\|x\|_{\gamma / 2}^{n, S}+2^{-1 / p} \sum_{k=1}^{n}\left(\begin{array}{l}
n \\
k
\end{array}\right)\left(\int_{0}^{1} d t t^{-1}\left(t^{(n-\gamma) / 2}\left\|S_{k t} x\right\|_{n}^{\prime}\right)^{p}\right)^{1 / p} .
$$

Now replacing $S$ by the semigroup $T$ where $T_{t}=S_{t} e^{-\omega t}$ one can choose $\omega$ sufficiently large that $t \mapsto\left\|T_{t}\right\|$ decreases exponentially as $t \rightarrow \infty$. Hence

$$
\int_{0}^{l} d t t^{-1}\left(t^{(n-\gamma) / 2}\left\|S_{k t} x\right\|_{n}^{\prime}\right)^{p} \leq k^{-(n-\gamma) p / 2} e^{k p \omega} \int_{0}^{\infty} d t t^{-1}\left(\left(t^{(n-\gamma) / 2}\left\|T_{t} x\right\|_{n}^{\prime}\right)^{p} .\right.
$$

Then since

$$
\left\|T_{t} x\right\|_{n}^{\prime} \leq \int_{t}^{\infty} d s\left\|(H+\omega I) T_{s} x\right\|_{n}^{\prime}
$$


one can use the semigroup property to obtain the bounds

$$
\begin{gathered}
\left(\int_{0}^{\infty} d t t^{-1}\left(t^{(n-\gamma) / 2}\left\|T_{t} x\right\|_{n}^{\prime}\right)^{p}\right)^{1 / p} \\
\leq\left(\int_{0}^{\infty} d t t^{-1}\left(t^{(n-\gamma) / 2} \int_{t / n}^{\infty} d s_{1} \ldots \int_{t / n}^{\infty} d s_{n}\left\|(H+\omega I)^{n} T_{s_{1}+\ldots+s_{n}} x\right\|_{n}^{\prime}\right)^{p}\right)^{1 / p} \\
=\left(\int _ { 0 } ^ { \infty } d t t ^ { - 1 } \left(t^{(n-\gamma) / 2}(2 t / n)^{n} \int_{1}^{\infty} d u_{1} \ldots\right.\right. \\
\left.\left.\leq(2 / n)^{n} \int_{1}^{\infty} d u_{1} \ldots \int_{1}^{\infty} d u_{n}\left\|(H+\omega I)^{n} T_{t\left(u_{1}^{2}+\ldots+u_{n}^{2}\right) / n} x\right\|_{n}^{\prime} u_{1} \ldots u_{n}\right)^{p}\right)^{1 / p} \\
\int_{1}^{\infty} d u_{n}\left(\int_{0}^{\infty} d t t^{-1}\left(t^{(3 n-\gamma) / 2}\left\|(H+\omega I)^{n} T_{t u^{2} / n} x\right\|_{n}^{\prime} u_{1} \ldots u_{n}\right)^{p}\right)^{1 / p},
\end{gathered}
$$

where $u^{2}=u_{1}^{2}+\cdots+u_{n}^{2}$. The last inequality is by the continuous version of the Minkowski inequality. At this stage we need a bound for $\left\|T_{t} x\right\|_{n}^{\prime}$.

LEMMA 3.3. There exists a $c>0$, depending on $n$, such that

$$
\left\|S_{t} x\right\|_{n}^{\prime} \leq c t^{-n / 2}\|x\|
$$

for all $t \in\langle 0,1]$ and $x \in \mathscr{X}$.

PROOF. For $a_{i} \in \mathfrak{g}$ let $\tilde{A}_{i}$ be the corresponding left invariant vector field on $G$ (see (3)). Let $K$ be the kernel of the semigroup $S$. By Varopoulos' parabolic Harnack inequality, [27, Theorem V.3.1], there exist $a, r>0$ such that for all $h \in G$ and $t \in\langle 0,1]$

$$
\sup _{h \in B_{r \sqrt{i}}^{\prime}(g)}\left|\left(\widetilde{A_{i_{1}}} \ldots \widetilde{A_{i_{n}}} K_{t}\right)(h)\right| \leq a t^{-n / 2} \inf _{h \in B_{r \sqrt{t}}^{\prime}(g)} K_{2 t}(h)
$$

for all $i_{1}, \ldots, i_{n} \in\left\{1, \ldots, d^{\prime}\right\}$ where

$$
B_{r}^{\prime}(g)=\left\{h \in G:\left|h g^{-1}\right|^{\prime} \leq r\right\} .
$$

(Varopoulos establishes this type of inequality for vector fields on a manifold and then the value of $a$ depends on the point $g$ in the manifold. But on a Lie group $a$ can be chosen to be independent of $g$ by translation invariance.) Therefore setting $h=g$ in (9) one has

$$
\left|\left(\widetilde{A_{i_{1}}} \ldots \widetilde{A_{i_{n}}} K_{t}\right)(g)\right| \leq a t^{-n / 2} K_{2 t}(g)
$$


for all $t \in\langle 0,1]$ and $g \in G$. But in addition one has the estimates

$$
K_{2 t}(g) \leq a^{\prime} t^{-D^{\prime} / 2} e^{-b\left(|g|^{\prime}\right)^{2} / t}
$$

valid for all $g \in G$ and $t \in\langle 0,1]$ and suitable $a^{\prime}, b>0$ by [22, Theorem IV.4.16], and the discussion on page 331 . So one obtains bounds

$$
\left|\left(\widetilde{A_{i_{1}}} \ldots \widetilde{A_{i_{n}}} K_{t}\right)(g)\right| \leq a t^{-n / 2-D^{\prime} / 2} e^{-b\left(|g|^{\prime}\right)^{2} / t}
$$

valid for all $g \in G$ and $t \in\langle 0,1]$ and suitable $a, b>0$. Next, for all $x \in \mathscr{X}$ and $t \in\langle 0,1]$

$$
\left\|A_{i_{1}} \ldots A_{i_{n}} S_{t} x\right\| \leq \int_{G} d g\left|\left(\widetilde{A_{i_{1}}} \ldots \widetilde{A_{i_{n}}} K_{t}\right)(g)\right|\|x\| .
$$

Using the bounds (10), the bounds (4) on the volume of the balls and splitting the integral into a sum of integrals over the sets

$$
\left\{g \in G: N \leq|g|^{\prime} t^{-1 / 2}<N+1\right\}
$$

gives bounds

$$
\left\|A_{i_{1}} \ldots A_{i_{n}} S_{t} x\right\| \leq c t^{-n / 2}\|x\|
$$

for a suitable $c>0$, uniform in $t \in\langle 0,1]$ and $x \in \mathscr{X}$.

We now continue Step 4 of the proof of Theorem 3.2.

By the previous lemma there is a $c>0$ such that

$$
\left\|T_{t} x\right\|_{n}^{\prime} \leq c t^{-n / 2}\|x\|
$$

for all $t \in\langle 0,1]$ and $x \in \mathscr{X}$ and since $T$ is exponential decreasing we may assume that these bounds hold for all $t \in\langle 0, \infty\rangle$ and $x \in \mathscr{X}$. Consequently

$$
\left\|(H+\omega I)^{n} T_{t} x\right\|_{n}^{\prime} \leq c(t / 2)^{-n / 2}\left\|(H+\omega I)^{n} T_{t / 2} x\right\| .
$$

Hence

$$
\begin{gathered}
\left(\int_{0}^{\infty} d t t^{-1}\left(t^{(n-\gamma) / 2}\left\|T_{t} x\right\|_{n}^{\prime}\right)^{p}\right)^{1 / p} \leq c(2 / n)^{n} \int_{1}^{\infty} d u_{1} \ldots \int_{1}^{\infty} d u_{n} \cdot \\
\cdot\left(\int_{0}^{\infty} d t t^{-1}\left(t^{(3 n-\gamma) / 2}\left(\frac{t u^{2}}{2 n}\right)^{-n / 2}\left\|(H+\omega I)^{n} T_{\frac{u^{2}}{2 n}} x\right\| u_{1} \ldots u_{n}\right)^{p}\right)^{1 / p} \\
=c(2 / n)^{n}(2 n)^{(3 n-\gamma) / 2}\left(\int_{1}^{\infty} d u_{1} \ldots \int_{1}^{\infty} d u_{n} u_{1} \ldots u_{n} u^{\gamma-3 n}\right)\|x\|_{\gamma / 2}^{n, \bar{H}}+\omega l
\end{gathered}
$$


But the integral over the $u$-variables is finite and $\|\cdot\|_{\gamma / 2}^{n, \bar{H}+\omega I}$ is equivalent to $\|\cdot\|_{\gamma / 2}^{n, S}$ by Theorem 3.1. Hence by combination of these estimates one concludes that

$$
\|x\|_{\gamma}^{(0, n)} \leq C\|x\|_{y / 2}^{n, S}
$$

for a suitable $C>0$ and all $x \in \mathscr{X}$. Thus $\mathscr{X}_{\gamma / 2}^{n, S}$ is continuously embedded in $\left(\mathscr{X}, \mathscr{X}_{n}^{\prime}\right)_{\gamma}$. The proof of Theorem 3.2 is complete.

Now we are prepared to prove Theorem 2.1. The proof relies on the characterizations of the interpolation spaces given by Theorem 3.2 and a number of standard results of interpolation theory.

PROOF OF THEOREM 2.1. First, one immediately obtains Statement II of Theorem 2.1 from Theorem 3.2. This follows because $\left(\mathscr{X}, \mathscr{X}_{n}^{\prime}\right)_{\gamma}=\mathscr{X}_{\gamma / 2}^{n, S}$. But $\mathscr{X}_{\gamma / 2}^{n, S}$ is independent of the choice of $n$, up to equivalence of norms by Theorem 3.1. Now we turn to the proof of Statements I, III and IV of Theorem 2.1.

Secondly, it follows from the argument used to prove [22, Lemma III.3.3] that there exists an $M>0$ such that

$$
\|x\|_{k}^{\prime} \leq \varepsilon^{n-k}\|x\|_{n}^{\prime}+M \varepsilon^{-k}\|x\|
$$

for all $\varepsilon>0, k \in\{1, \ldots, n-1\}$ and all $x \in \mathscr{X}_{n}^{\prime}$. Hence by choosing $\varepsilon$ appropriately one obtains bounds

$$
\|x\|_{k}^{\prime} \leq(1+M)\|x\|^{1-k / n}\left(\|x\|_{n}^{\prime}\right)^{k / n} .
$$

Therefore $\mathscr{X}_{k}^{\prime}$ belongs to the class $J\left(k / n, \mathscr{X}, \mathscr{X}_{n}^{\prime}\right)$ by [25, Lemma 1.10.1]. Hence by the reiteration theorem, [6, Proposition 3.2.19],

$$
\left(\mathscr{X}, \mathscr{X}_{n}^{\prime}\right)_{\gamma} \subseteq\left(\mathscr{X}_{k}^{\prime}, \mathscr{X}_{n}^{\prime}\right)_{\gamma-k}
$$

and the embedding is continuous.

It now follows that

$$
\left(\mathscr{X}, \mathscr{X}_{n+k}^{\prime}\right)_{\gamma+k} \subseteq\left(\mathscr{X}_{k}^{\prime}, \mathscr{X}_{n+k}^{\prime}\right)_{\gamma} \subseteq \mathscr{X}_{k}^{\prime}
$$

Next let $M_{l}$ denote an $l$ th order monomial in the operators $A_{1}, \ldots, A_{d^{\prime}}$ where $l \leq k$. Then $M_{l}$ maps $\mathscr{X}_{k}^{\prime}$ continuously into $\mathscr{X}$ and $\mathscr{X}_{n+k}^{\prime}$ continuously into $\mathscr{X}_{n}^{\prime}$. So by interpolation theory, [6, Theorem 3.2.23], $M_{l}$ maps $\left(\mathscr{X}_{k}^{\prime}, \mathscr{X}_{n+k}^{\prime}\right)_{\gamma}$ continuously into $\left(\mathscr{X}, \mathscr{X}_{n}^{\prime}\right)_{\gamma}$. Therefore $\left(\mathscr{X}_{k}^{\prime}, \mathscr{X}_{n+k}^{\prime}\right)_{\gamma}$ is continuously embedded in $\left(\mathscr{X}, \mathscr{X}_{n}^{\prime}\right)_{\gamma ; k}$ and from the previous observation one then has the continuous embedding

$$
\left(\mathscr{X}, \mathscr{X}_{n+k}^{\prime}\right)_{\gamma+k} \subseteq\left(\mathscr{X}, \mathscr{X}_{n}^{\prime}\right)_{\gamma ; k}
$$


The proof of the converse inclusion is based on the estimate (2). A $k$-fold iteration of this inequality gives bounds

$$
\omega_{x}^{(n+k)}(t) \leq M^{k} t^{k} \sup _{1 \leq i_{1}, \ldots, i_{k} \leq d^{\prime}} \omega_{A_{i_{1}} \ldots A_{i_{k}} x}^{(n)}((1+\varepsilon) t)
$$

for all $t \in\langle 0,1]$ and $\varepsilon \in\langle 0,1]$. Hence by taking $\varepsilon=1$ one immediately finds bounds

$$
\|x\|_{\gamma+k}^{n+k, \omega} \leq C_{k}\left(\|x\|+\sup \left\|M_{k} x\right\|_{\gamma}^{n, \omega}\right)
$$

where the supremum is over all $k$ th order monomials $A_{1}, \ldots, A_{d^{\prime}}$. But since $\|\cdot\|_{\gamma}^{n, \omega}$ is equivalent to $\|\cdot\|_{\gamma}^{(0, n)}$ by Theorem 3.2 this establishes the continuous embedding

$$
\left(\mathscr{X}, \mathscr{X}_{n}^{\prime}\right)_{\gamma ; k} \subseteq\left(\mathscr{X}, \mathscr{X}_{n+k}^{\prime}\right)_{\gamma+k}
$$

and completes the proof of Statement III of Theorem 2.1.

Next, if $x \in \mathscr{X}_{\gamma}^{(0, n)}$ then

$$
\|x\|_{\gamma}^{(0, n)} \leq C\left(\|x\|+\sup \left\|M_{k} x\right\|_{\gamma-k}^{n-k, \omega}\right)
$$

by the foregoing estimates. But if $x \in \mathscr{X}_{k}^{\prime}$ one also has

$$
\kappa_{M_{k} x}^{(0, n-k)}(t) \leq \inf \left\{\left\|M_{k} x_{k}\right\|+t^{n-k}\left\|M_{k} x_{n}\right\|_{n-k}^{\prime}: x=x_{k}+x_{n}, x_{n} \in \mathscr{X}_{n}^{\prime}\right\} .
$$

Consequently

$$
\begin{aligned}
\kappa_{M_{k} x}^{(0, n-k)}(t) & \leq \inf \left\{\left\|x_{k}\right\|_{k}^{\prime}+t^{n-k}\left\|x_{n}\right\|_{n}^{\prime}: x=x_{0}+x_{n}, x_{n} \in \mathscr{X}_{n}^{\prime}\right\} \\
& =\kappa_{x}^{(k, n)}(t)
\end{aligned}
$$

and this gives bounds

$$
\left\|M_{k} x\right\|_{y-k}^{(0, n-k)} \leq\|x\|_{\gamma-k}^{(k, n)} .
$$

Since $\|\cdot\|_{\gamma-k}^{n-k, \omega}$ is equivalent to $\|\cdot\|_{\gamma-k}^{(0, n-k)}$ a combination of (12) and (13) gives estimates

$$
\|x\|_{\gamma}^{(0, n)} \leq c\|x\|_{\gamma-k}^{(k, n)}
$$

for all $x \in \mathscr{X}_{k}^{\prime}$. So we have established the continuous embedding

$$
\left(\mathscr{X}_{k}^{\prime}, \mathscr{X}_{n}^{\prime}\right)_{\gamma-k} \subseteq\left(\mathscr{X}, \mathscr{X}_{n}^{\prime}\right)_{\gamma} .
$$

But (11) establishes the converse inclusion and hence we have now deduced Statement I of Theorem 2.1.

Finally, Statement IV can be deduced from Statement II. To this end let $S$ denote the semigroup generated by the closure of the sublaplacian

$$
H=-\sum_{i=1}^{d^{\prime}} A_{i}^{2}
$$


then it follows from [22, Corollary IV.4.17], that $S_{t} \mathscr{X} \subseteq \mathscr{X}_{\infty}$. Therefore $S_{t} \mathscr{X}_{n}^{\prime} \subseteq$ $\mathscr{X}_{n}^{\prime}$ for each $n$. Consequently, $S_{t}\left(\mathscr{X}, \mathscr{X}_{n}^{\prime}\right)_{\gamma} \subseteq\left(\mathscr{X}, \mathscr{X}_{n}^{\prime}\right)_{\gamma}$. Next by continuity one has bounds

$$
\left\|S_{t}\right\| \leq M e^{\omega t}
$$

for suitable $M \geq 1$ and $\omega \geq 0$. Therefore it follows from the identification

$$
\left(\mathscr{X}, \mathscr{X}_{n}^{\prime}\right)_{\gamma}=\mathscr{X}_{\gamma / 2}^{n . S}
$$

that $S$ restricted to $\left(\mathscr{X}, \mathscr{X}_{n}^{\prime}\right)_{\gamma}$ satisfies similar bounds. Now we argue that this restriction is strongly continuous, with respect to $\|\cdot\|_{\gamma}^{(0, n)}$. But because of the semigroup property and the foregoing boundedness property it suffices to establish continuity at the origin on a dense subset of $\left(\mathscr{X}, \mathscr{X}_{n}^{\prime}\right)_{\gamma}$.

Next remark that if $p<\infty$ then $\mathscr{X}_{n}^{\prime}$ is norm-dense in $\left(\mathscr{X}, \mathscr{X}_{n}^{\prime}\right)_{\gamma}$ by general interpolation theory. Hence $\mathscr{X}_{n_{1}}^{\prime}$ is norm-dense in $\left(\mathscr{X}, \mathscr{X}_{n}^{\prime}\right)_{\gamma}$ for all $n_{1} \geq n$ by Statement II of the Theorem. Then for $x \in \mathscr{X}_{n+2}^{\prime}$ one has

$$
\begin{aligned}
\left\|S_{t} x-x\right\|_{\gamma}^{(0, n)} & \leq \int_{0}^{t} d s\left\|S_{s} H x\right\|_{\gamma}^{(0, n)} \\
& \leq c t\|H x\|_{\gamma}^{(0, n)} \\
& \leq C t\|H x\|_{n}^{\prime}
\end{aligned}
$$

for all $x \in \mathscr{X}_{n+2}^{\prime}$, all $t \in\langle 0,1]$, and suitable $C, c>0$. Therefore $S$ is strongly continuous on the norm-dense subspace $\mathscr{X}_{n+2}^{\prime}$ of $\left(\mathscr{X}, \mathscr{X}_{n}^{\prime}\right)_{\gamma}$ and consequently it is strongly continuous on $\left(\mathscr{X}, \mathscr{X}_{n}^{\prime}\right)_{\gamma}$.

Finally, since $S_{t} \mathscr{X} \subseteq \mathscr{X}_{\infty}$ it follows that $\mathscr{X}_{\infty}$ is norm-dense in $\left(\mathscr{X}, \mathscr{X}_{n}^{\prime}\right)_{\gamma}$ and the proof of Theorem 2.1 is complete.

It should be noted that the last statement of the theorem, the density of $\mathscr{X}_{\infty}$, is not always valid if $p=\infty$. In addition the restriction of the semigroup $S$ to the interpolation space is not always continuous for the limiting value of $p$. Counterexamples are easily constructed. For example, if we start with the left regular representation $L$ on $\mathscr{X}=L_{\infty}(\mathbb{R})$ and $f \in\left(\mathscr{X}, \mathscr{X}_{2}\right)_{1}$ is as in [24, Example V.4.3.1], then the restriction of $L$ to $\left(\mathscr{X}, \mathscr{X}_{2}\right)_{1}$ is not continuous at $f$ by [6, Proposition 3.4.13]. The fact that $L$ is not strongly continuous is not important, by the next corollary.

COROLlARY 3.4. Let $(\mathscr{X}, G, U)$ be a weakly* continuous representation, $\widetilde{\mathscr{X}}$ the subspace on which $U$ acts strongly continuously and $\tilde{U}$ the restriction of $U$ to $\widetilde{X}$. If $n>\gamma>0$ then

$$
\left(\mathscr{X}, \mathscr{X}_{n}^{\prime}\right)_{\gamma}=\left(\widetilde{\mathscr{X}}, \widetilde{\mathscr{X}}_{n}^{\prime}\right)_{\gamma}
$$

and the norms are equivalent, where $\left(\widetilde{\mathscr{X}}, \widetilde{\mathscr{X}}_{n}^{\prime}\right)_{\gamma}$ is the Lipschitz space with respect to the strongly continuous representation $(\widetilde{\mathscr{X}}, G, \widetilde{U})$. 
PROOF. Let $S$ be the holomorphic semigroup generated by the weak ${ }^{*}$-weak ${ }^{*}$-closure $\bar{H}$ of the sublaplacian. Since $\widetilde{\mathscr{X}}$ is the norm closure of $\mathscr{X}_{\infty}$ and $S_{t}$ maps $\mathscr{X}$ into $\mathscr{X}_{\infty} \subseteq D(\bar{H})$, it follows that $\widetilde{\mathscr{X}}$ is the subspace of $\mathscr{X}$ on which $S$ acts strongly continuously. Let $\widetilde{S}$ be the restriction of $S$ to $\widetilde{\mathscr{X}}$. Let $\widehat{S}$ be the strongly continuous semigroup generated by the strong closure of the Laplacian in the representation $(\widetilde{\mathscr{X}}, G, \widetilde{U})$. Then the infinitesimal generator of $\widetilde{S}$ is an extension of the infinitesimal generator of $\widehat{S}$, so $\widetilde{S}_{t}=\widehat{S}_{t}$ for all $t \geq 0$. Then by Theorems 3.1 and 3.2

$$
\left(\mathscr{X}, \mathscr{X}_{n}^{\prime}\right)_{\gamma}=\mathscr{X}_{\gamma / 2}^{n, S}=\mathscr{X}_{\gamma / 2}^{n, \tilde{S}}=\left(\widetilde{\mathscr{X}}, \widetilde{\mathscr{X}}_{n}^{\prime}\right)_{\gamma}
$$

and the norms are equivalent.

If $a_{1}, \ldots, a_{d^{\prime}}$ is not a full vector space basis of $\mathfrak{g}$ then the subspaces $\mathscr{X}_{n}^{\prime}$ need not be invariant under the representation $U$ (see Example 7.4 below). Also the interpolation spaces are not $U$-invariant in general. Since, however,

$$
\mathscr{X}_{n r}^{\prime} \subseteq \mathscr{X}_{n} \subseteq \mathscr{X}_{n}^{\prime},
$$

and $\mathscr{X}_{n}$ is $U$-invariant, it follows that

$$
U \mathscr{X}_{n r}^{\prime} \subseteq \mathscr{X}_{n}^{\prime}
$$

Nevertheless if $S$ is the continuous semigroup generated by the closure of a secondorder subelliptic operator $H$ constructed from the representatives $A_{1}, \ldots, A_{d^{\prime}}$ then $S$ does leave the interpolation spaces invariant by the argument used above for the semigroup generated by the sublaplacian. Moreover, if $p \in[1, \infty)$ the action of each of the $S$ is continuous. In Theorem 5.1 we shall prove that $S$ is a holomorphic semigroup on each of the Lipschitz spaces.

\section{Regularity properties}

The Lipschitz spaces can be exploited to yield regularity properties of the sublaplacian used in Theorem 3.2. These then provide perturbation estimates which allow one to extend the results of Section 3, and the regularity properties, to second-order subelliptic operators of the type discussed in the introduction. These extensions will be described in detail in the subsequent sections.

First we establish regularity on the Lipschitz spaces.

THEOREM 4.1. Let

$$
H=-\sum_{i=1}^{d^{\prime}} A_{i}^{2}+\omega I
$$

where $\omega$ is chosen large enough that the semigroup generated by the closure $\bar{H}$ of $H$ is uniformly bounded. 
I. If $n>\gamma>\alpha>0$ then

$$
\left(\mathscr{X}, \mathscr{X}_{n}^{\prime}\right)_{\gamma}=\left\{x \in D\left(\bar{H}^{\alpha / 2}\right): \bar{H}^{\alpha / 2} x \in\left(\mathscr{X}, \mathscr{X}_{n}^{\prime}\right)_{\gamma-\alpha}\right\}
$$

and the norms $x \mapsto\|x\|_{\gamma}^{(0, n)}$ and $x \mapsto\left\|\bar{H}^{\alpha / 2} x\right\|_{\gamma-\alpha}^{(0, n)}+\|x\|_{\gamma-\alpha}^{(0, n)}$ are equivalent.

II. If $n>\gamma>0$ and $k \in \mathbb{N}$ then

$$
\left(\mathscr{X}, \mathscr{X}_{n}^{\prime}\right)_{\gamma ; k}=\left\{x \in D\left(\bar{H}^{k / 2}\right): \bar{H}^{k / 2} x \in\left(\mathscr{X}, \mathscr{X}_{n}^{\prime}\right)_{\gamma}\right\}
$$

and the norms $x \mapsto\|x\|_{\gamma ; k}^{(0, n)}$ and $x \mapsto\left\|\bar{H}^{k / 2} x\right\|_{\gamma}^{(0, n)}+\|x\|_{\gamma}^{(0, n)}$ are equivalent.

In the proof of this theorem we need the following lemma.

LEMMA 4.2. Let $S$ be a bounded holomorphic semigroup with generator $H$. For $\alpha \in\langle 0,1\rangle$ let $P^{\alpha}$ be the semigroup generated by $H^{\alpha}$. If $n>\max (\gamma, \gamma /(2 \alpha))$ then

$$
\mathscr{X}_{\gamma}^{n, S}=\mathscr{X}_{\gamma /(2 \alpha)}^{n, P^{\alpha}}
$$

and the norms $\|\cdot\|_{\gamma}^{n, s}$ and $\|\cdot\|_{\gamma /(2 \alpha)}^{n, P^{\alpha}}$ are equivalent.

PROOF. We may assume that $S$ is exponentially decreasing.

Then $\mathscr{X}_{\gamma}^{n, S}=\left(\mathscr{X}, D\left(H^{n}\right)\right)_{\gamma / n, p ; \mathrm{K}}$ by Theorem 3.1 and the norms are equivalent. Similarly $\mathscr{X}_{\gamma / \alpha}^{n, P^{\alpha}}=\left(\mathscr{X}, D\left(\left(H^{\alpha}\right)^{n}\right)\right)_{\gamma /(n \alpha), p ; \mathrm{K}}$ with equivalent norms. Clearly $D\left(H^{n \alpha}\right)$ is an intermediate space of $\mathscr{X}$ and $D\left(H^{n}\right)$. But by [22, Lemmas II.2.5 and II.3.3] there exists an $M>0$ such that

$$
\left\|H^{n \alpha} x\right\| \leq \varepsilon^{n(1-\alpha)}\left\|H^{n} x\right\|+M \varepsilon^{-n \alpha}\|x\|
$$

for all $\varepsilon>0$ and $x \in D\left(H^{n}\right)$. (The restriction $\varepsilon \leq 1$ in [22, Lemma II.2.5] can be removed if $H$ is invertible and this is the case since $S$ is exponentially decreasing.) Therefore by choosing $\varepsilon$ appropriately one obtains bounds

$$
\left\|H^{n \alpha} x\right\| \leq(M+1)\|x\|^{1-\alpha}\left\|H^{n} x\right\|^{\alpha}
$$

for all $x \in D\left(H^{n}\right)$. So $D\left(H^{n \alpha}\right)$ belongs to the class $J\left(\alpha, \mathscr{X}, D\left(H^{n}\right)\right)$ by [25, Lemma 1.10.1].

For $x \in \mathscr{X}$ and $t>0$ let

$$
\kappa_{x}(t)=\inf \left\{\left\|x_{0}\right\|+t\left\|H^{n} x_{n}\right\|: x=x_{0}+x_{n}, x_{0} \in \mathscr{X} \text { and } x_{n} \in D\left(H^{n}\right)\right\} .
$$

By [22, Lemma II.3.3] and the holomorphy of $S$ it follows that there exists a $c>0$ such that

$$
\left\|H^{1-\alpha} S_{t} x\right\| \leq c t^{-(1-\alpha)}\|x\|
$$


for all $t \in\langle 0,1]$ and $x \in \mathscr{X}$. But since $S$ is exponentially decreasing, we may assume that (14) is valid for all $t>0$. Now let $x \in D\left(H^{n \alpha}\right)$ and $t>0$. Since $S$ is a holomorphic semigroup,

$$
\kappa_{x}(t) \leq\left\|\left(I-S_{t^{1 / n}}\right)^{n} x\right\|+t\left\|H^{n}\left(I-\left(I-S_{t^{1 / n}}\right)^{n}\right) x\right\| .
$$

We consider the two terms separately. First, one has

$$
\begin{aligned}
\left\|\left(I-S_{t^{1 / n}}\right)^{n} x\right\| & \leq \int_{0}^{t^{1 / n}} d s_{1} \ldots \int_{0}^{t^{1 / n}} d s_{n}\left\|H^{1-\alpha} S_{s_{1}} \ldots H^{1-\alpha} S_{s_{n}} H^{n \alpha} x\right\| \\
& \leq c^{n} \int_{0}^{t^{1 / n}} d s_{1} \ldots \int_{0}^{t^{1 / n}} d s_{n} s_{1}^{-(1-\alpha)} \ldots s_{n}^{-(1-\alpha)}\left\|H^{n \alpha} x\right\| \\
& =c^{n} \alpha^{-n} t^{\alpha}\left\|H^{n \alpha} x\right\| .
\end{aligned}
$$

Secondly,

$$
t\left\|H^{n}\left(I-\left(I-S_{t}{ }^{1 / n}\right)^{n}\right) x\right\| \leq \sum_{k=1}^{n}\left(\begin{array}{l}
n \\
k
\end{array}\right) t\left\|H^{n} S_{k t^{1 / n}} x\right\| .
$$

Let $k \in\{1, \ldots, n\}$. Then by a similar reasoning to the above

$$
t\left\|H^{n} S_{k t^{1 / n} x}\right\| \leq t c^{n}\left(k t^{1 / n} / n\right)^{-n(1-\alpha)}\left\|H^{n \alpha} x\right\|=c^{n}(n / k)^{n(1-\alpha)} t^{\alpha}\left\|H^{n \alpha} x\right\| .
$$

So $D\left(H^{n \alpha}\right)$ belongs to the class $K\left(\alpha, \mathscr{X}, D\left(H^{n}\right)\right)$. Then by the reiteration theorem ([6, Theorem 3.2.20]) it follows that $\left(\mathscr{X}, D\left(H^{n}\right)\right)_{\gamma / n, p ; \mathrm{K}}=\left(\mathscr{X}, D\left(H^{n \alpha}\right)\right)_{\gamma /(n \alpha), p ; \mathrm{K}}$ and the norms are equivalent.

PROOF OF THEOREM 4.1. Let $k \in \mathbb{N}$ and $\beta \in\langle 0,2\rangle$ be such that $\alpha=k \beta$ and let $q \in \mathbb{N}$ with $q>\gamma / \beta$. Further let $P$ be the semigroup generated by $\bar{H}^{\beta / 2}$. Then by Lemma 4.2 and [6, Theorem 3.4.6] one obtains

$$
\begin{aligned}
\left(\mathscr{X}, \mathscr{X}_{n}^{\prime}\right)_{\gamma} & =\mathscr{X}_{\gamma}^{n, S}=\mathscr{X}_{\gamma / \beta}^{q, P} \\
& =\left\{x \in D\left(\left(\bar{H}^{\beta / 2}\right)^{k}\right): \bar{H}^{k \beta / 2} x \in \mathscr{X}_{\gamma / \beta-k}^{q, P}\right\} \\
& =\left\{x \in D\left(\bar{H}^{\alpha / 2}\right): \bar{H}^{\alpha / 2} x \in\left(\mathscr{X}, \mathscr{X}_{n}^{\prime}\right)_{\gamma-\alpha}\right\}
\end{aligned}
$$

Also the norms are equivalent. This proves Statement I.

Statement II follows immediately from Statement I and Theorem 2.1.III.

Next we derive some additional regularity properties for the interpolation spaces, comparisons between the Lipschitz spaces, and regularity of the underlying representation $(\mathscr{X}, G, U)$. Note that the statements of the following proposition indicate that $\|\cdot\|_{\gamma}^{(0, n)}$ behaves as a $C^{\gamma}$-norm and $\bar{H}^{\alpha / 2}$ as a fractional derivative of order $\alpha$. 
Until now we have omitted all reference to $p$ in the notation of the Lipschitz space $\left(\mathscr{X}_{n_{1}}^{\prime}, \mathscr{X}_{n_{2}}^{\prime}\right)_{\gamma}$, but for the next two propositions the value of $p$ is important. Therefore we denote the Lipschitz space by $\left(\mathscr{X}_{n_{1}}^{\prime}, \mathscr{X}_{n_{2}}^{\prime}\right)_{(\gamma, p)}$ and its norm by $\|\cdot\|_{(\gamma, p)}^{\left(n_{1}, n_{2}\right)}$.

Next we introduce the concept of a Banach space of order $\gamma$, where $\gamma \geq 0$. The space $\mathscr{X}$ is the only space of order 0 . If $0<\gamma<n$ then the spaces of order $\gamma$ are the Lipschitz spaces $\left(\mathscr{X}, \mathscr{X}_{n}^{\prime}\right)_{(\gamma, p)}$ with $p \in[1, \infty]$ and the spaces $D\left(\bar{H}^{\gamma / 2}\right)$ with

$$
H=-\sum_{i=1}^{d^{\prime}} A_{i}^{2}+\omega I
$$

where $\omega$ is sufficiently large that the semigroup generated by the closure $\bar{H}$ of $H$ is uniformly bounded. Moreover, if $\gamma \in \mathbb{N}$ then the $C^{n}$-subspace $\mathscr{X}_{\gamma}^{\prime}$ is also defined to be of order $\gamma$.

PROPOSITION 4.3. Let

$$
H=-\sum_{i=1}^{d^{\prime}} A_{i}^{2}+\omega I
$$

where $\omega$ is chosen large enough that the semigroup generated by the closure $\bar{H}$ of $H$ is uniformly bounded.

I. If $0 \leq \gamma_{1}<\gamma_{2}<\gamma_{3}$ and $\mathscr{Y}_{i}$ is a Banach space of order $\gamma_{i}$ with norm $\|\cdot\|_{\gamma_{i}}$ then there exists a $c>0$ such that

$$
\|x\|_{\gamma_{2}} \leq \varepsilon^{\gamma_{3}-\gamma_{2}}\|x\|_{\gamma_{3}}+c \varepsilon^{-\left(\gamma_{2}-\gamma_{1}\right)}\|x\|_{\gamma_{1}}
$$

for all $x \in \mathscr{Y}_{3}$ and $\varepsilon>0$.

II. If $p=\infty$ and $n>\gamma>0$ then there exists a $c>0$ such that

$$
\|x\|_{(\gamma, \infty)}^{(0, n)} \leq c\left(\left\|\bar{H}^{\gamma / 2} x\right\|+\|x\|\right)
$$

for all $x \in D\left(\bar{H}^{\gamma / 2}\right)$.

PROOF. We only prove the case $\gamma_{1} \neq 0$; the case $\gamma_{1}=0$ can be proved similarly. First we prove (15) for the Lipschitz spaces. By Theorem 2.1 we may assume that there are $n>\gamma_{3}$ and $p_{1}, p_{2}, p_{3} \in[1, \infty]$ such that $\mathscr{Y}_{i}=\left(\mathscr{X}, \mathscr{X}_{n}^{\prime}\right)_{\left(\gamma_{i}, p_{i}\right)}$. Then by the reiteration theorem, [6, Theorem 3.2.20 and Corollary 3.2.17],

$$
\left(\mathscr{Y}_{1}, \mathscr{Y}_{3}\right)_{\left(\gamma_{2}-\gamma_{1}\right) /\left(\gamma_{3}-\gamma_{1}\right), 1 ; \mathrm{K}}=\left(\mathscr{X}, \mathscr{X}_{n}^{\prime}\right)_{\gamma_{2} / n, 1 ; \mathrm{K}} \subseteq \mathscr{Y}_{2},
$$

where the inclusion is by [6, Corollary 3.2.13]. So by [25, Lemma 1.10.1(a)] there exists a $c>0$ such that

$$
\|x\|_{\gamma_{2}} \leq c\|x\|_{\gamma_{1}}^{\left(\gamma_{3}-\gamma_{2}\right) /\left(\gamma_{3}-\gamma_{1}\right)}\|x\|_{\gamma_{3}}^{\left(\gamma_{2}-\gamma_{1}\right) /\left(\gamma_{3}-\gamma_{1}\right)}
$$


for all $x \in \mathscr{Y}_{3}$. Hence by the Hölder inequality

$$
\|x\|_{\gamma_{2}} \leq c \frac{\left(\gamma_{2}-\gamma_{1}\right)}{\left(\gamma_{3}-\gamma_{1}\right)} \delta^{\left(\gamma_{3}-\gamma_{2}\right) /\left(\gamma_{3}-\gamma_{1}\right)}\|x\|_{\gamma_{3}}+c \frac{\left(\gamma_{3}-\gamma_{2}\right)}{\left(\gamma_{3}-\gamma_{1}\right)} \delta^{-\left(\gamma_{2}-\gamma_{1}\right) /\left(\gamma_{3}-\gamma_{1}\right)}\|x\|_{\gamma_{1}}
$$

for all $\delta>0$. Inequality (15) follows for the Lipschitz spaces by choosing $\varepsilon$ to be an appropriate multiple of $\delta^{1 /\left(\gamma_{3}-\gamma_{1}\right)}$.

The general case, and also part II of the proposition, follows from the inequalities

$$
\begin{aligned}
\|x\|_{(m, \infty)}^{(0, n)} & \leq c_{1}\|x\|_{m}^{\prime} \\
\|x\|_{m}^{\prime} & \leq c_{2}\|x\|_{(m, 1)}^{(0, n)} \\
\|x\|_{(m, \infty)}^{(0, n)} & \leq c_{3}\left(\left\|\bar{H}^{\gamma / 2} x\right\|+\|x\|\right) \\
\left\|\bar{H}^{\gamma / 2} x\right\|+\|x\| & \leq c_{4}\|x\|_{(m, 1)}^{(0, n)}
\end{aligned}
$$

for constants $c_{1}, \ldots, c_{4}>0$ and all $x \in \mathscr{X}_{m}^{\prime}, x \in\left(\mathscr{X}, \mathscr{X}_{n}^{\prime}\right)_{(m, 1)}, x \in D\left(\bar{H}^{\gamma / 2}\right)$ and $x \in\left(\mathscr{X}, \mathscr{X}_{n}^{\prime}\right)_{(\gamma, 1)}$, respectively, where $m<n$ and $\gamma<n$.

By an $m$-fold iteration of inequality (2) we obtain

$$
\omega_{x}^{(n)}(t) \leq M t^{m} \sup _{i_{1}, \ldots, i_{m} \in\left\{1, \ldots, d^{\prime}\right\}} \omega_{A_{i_{1}} \ldots A_{i_{m} x}}^{(n-m)}(2 t)
$$

for all $x \in \mathscr{X}_{m}^{\prime}$ and $t \in\langle 0,1]$. Using the inequality

$$
\omega_{y}^{(n-m)}(t) \leq c\|y\|
$$

for some $c>0$, uniform in $y \in \mathscr{X}$ and $t \in\langle 0,1]$ we then find that

$$
\omega_{x}^{(n)}(t) \leq c M t^{m}\|x\|_{m}^{\prime}
$$

for all $x \in \mathscr{X}_{m}^{\prime}$ and $t \in\langle 0,1]$. So

$$
\|x\|_{(m, \infty)}^{(0, n)} \leq c M\|x\|_{m}^{\prime}
$$

and (16) is proved.

In a similar way to the proof of [22, Lemma III.3.3] it follows that there exists a $c>0$ such that

$$
\|x\|_{m}^{\prime} \leq \varepsilon^{n-m}\|x\|_{n}^{\prime}+c \varepsilon^{-m}\|x\|
$$

for all $x \in \mathscr{X}_{n}^{\prime}$ and $\varepsilon>0$. Minimizing over $\varepsilon$ gives bounds

$$
\|x\|_{m}^{\prime} \leq(1+c)\|x\|^{1-m / n}\left(\|x\|_{n}^{\prime}\right)^{m / n}
$$


and so again by [25, Lemma 1.10.1(a)] it follows that

$$
\left(\mathscr{X}, \mathscr{X}_{n}^{\prime}\right)_{(m, 1)} \subseteq \mathscr{X}_{m}^{\prime}
$$

Now inequality (17) follows from the closed graph theorem.

By [25, Theorem 1.15.2(d)]

$$
\left(\mathscr{X}, D\left(\bar{H}^{n}\right)\right)_{\gamma /(2 n), 1 ; \mathrm{K}} \subseteq D\left(\bar{H}^{\gamma / 2}\right) \subseteq\left(\mathscr{X}, D\left(\bar{H}^{n}\right)\right)_{\gamma /(2 n), \infty ; \mathrm{K}} .
$$

But by Theorems 3.1 and 3.2

$$
\left(\mathscr{X}, D\left(\bar{H}^{n}\right)\right)_{\gamma /(2 n), p ; \mathrm{K}}=\left(\mathscr{X}, \mathscr{X}_{n}^{\prime}\right)_{(\gamma, p)} .
$$

Then inequalities (18) and (19) follow by the closed graph theorem and the proposition is proved.

The next proposition shows that the semigroup $S$ has the behaviour expected for a semigroup generated by a second-order operator.

PROPOSITION 4.4. Let

$$
H=-\sum_{i=1}^{d^{\prime}} A_{i}^{2}+\omega I
$$

where $\omega$ is chosen large enough that the semigroup $S$ generated by the closure $\bar{H}$ of $H$ is uniformly bounded. If $0 \leq \gamma_{1}<\gamma_{2}$ and $\mathscr{Y}_{i}$ is a Banach space of order $\gamma_{i}$ with norm $\|\cdot\|_{\gamma_{i}}$ then there exists a $c>0$ such that

$$
\left\|S_{t} x\right\|_{\gamma_{2}} \leq c t^{-\left(\gamma_{2}-\gamma_{1}\right) / 2}\|x\|_{\gamma_{1}}
$$

for all $x \in \mathscr{Y}_{1}$ and $t \in\langle 0,1]$.

ProOF. As outlined in the proof of Proposition 4.3 we only need to consider the case $\gamma_{1}>0$ and Lipschitz spaces. Furthermore, we may assume that the semigroup is exponentially decreasing.

First, if $n>\gamma+\delta>\gamma>0$ and $p \in[1, \infty]$ then by Theorems 4.1.I and 3.2 there exists a $c_{1}>0$ such that

$$
\|x\|_{(\gamma+\delta, p)}^{(0, n)} \leq c_{1}\left\|\widehat{H}^{\delta / 2} x\right\|_{(\gamma / 2, p)}^{n, S}
$$

for all $x \in \mathscr{X}_{\infty}$. Since $S$ is a holomorphic semigroup there also exists a $c_{2}>0$ such that

$$
\left\|\bar{H}^{\delta / 2} S_{t} x\right\| \leq c_{2} t^{-\delta / 2}\|x\|
$$


for all $t \in\langle 0,1]$ and $x \in \mathscr{X}$. So

$$
\left\|S_{t} x\right\|_{(\gamma+\delta, p)}^{(0, n)} \leq c_{1} c_{2} t^{-\delta / 2}\|x\|_{(\gamma / 2, p)}^{n, S} \leq c_{3} t^{-\delta / 2}\|x\|_{(\gamma, p)}^{(0, n)}
$$

for all $t \in\langle 0,1]$ and $x \in\left(\mathscr{X}, \mathscr{X}_{n}^{\prime}\right)_{(\gamma, p)}$.

Secondly, if $0<\gamma<n$ and $p \in[1, \infty]$ then there exists an $M>0$ such that

$$
\left\|S_{t} x\right\|_{(\gamma, p)}^{(0, n)} \leq M\|x\|_{(\gamma, p)}^{(0, n)}
$$

for all $x \in\left(\mathscr{X}, \mathscr{X}_{n}^{\prime}\right)_{(\gamma, p)}$ and $t \in\langle 0,1]$. This follows again from the identification $\left(\mathscr{X}, \mathscr{X}_{n}^{\prime}\right)_{(\gamma, p)}=\mathscr{X}_{(\gamma / 2, p)}^{n, S}$.

Finally, if $0<\gamma_{1}<\gamma_{2}<n-1$ and $p_{1}, p_{2} \in[1, \infty]$ then by Proposition 4.3 there exists a $c_{1}>0$ such that

$$
\|x\|_{\left(\gamma_{2}, p_{2}\right)}^{(0, n)} \leq \varepsilon\|x\|_{\left(\gamma_{2}+1, p_{1}\right)}^{(0, n)}+c_{1} \varepsilon^{-\left(\gamma_{2}-\gamma_{1}\right)}\|x\|_{\left(\gamma_{1}, p_{1}\right)}^{(0, n)}
$$

for all $x \in\left(\mathscr{X}, \mathscr{X}_{n}^{\prime}\right)_{\left(\gamma_{2}+1, p_{1}\right)}$ and $\varepsilon>0$. So

$$
\left\|S_{t} x\right\|_{\left(\gamma_{2}, p_{2}\right)}^{(0, n)} \leq c_{3} \varepsilon t^{-\left(\gamma_{2}+1-\gamma_{1}\right) / 2}\|x\|_{\left(\gamma_{1}, p_{1}\right)}^{(0, n)}+c_{1} M \varepsilon^{-\left(\gamma_{2}-\gamma_{1}\right)}\|x\|_{\left(\gamma_{1}, p_{1}\right)}^{(0, n)}
$$

for all $x \in\left(\mathscr{X}, \mathscr{X}_{n}^{\prime}\right)_{\left(\gamma_{1}, p_{1}\right)}$ and $t \in\langle 0,1]$. The proposition follows by setting $\varepsilon=\sqrt{t}$.

\section{Subelliptic operators}

In this section we prove that all the results derived in the previous sections for the sublaplacian are stable under the addition of a complex first-order perturbation.

We now consider operators of the form

$$
H=-\sum_{i, j=1}^{d^{\prime}} c_{i j} A_{i} A_{j}+\sum_{i=1}^{d^{\prime}} c_{i} A_{i}+c_{0} I
$$

where $C=\left(c_{i j}\right)$ is a real-valued strictly positive-definite matrix and $c_{0}, c_{1}, \ldots, c_{d^{\prime}} \in$ C. Since $C$ can be diagonalized by a real-valued non-singular matrix it follows that by a change of algebraic basis one may assume that $c_{i j}=\delta_{i j}$. Therefore one can effectively restrict attention to operators $H=H_{0}+H_{1}$ where

$$
H_{0}=-\sum_{i=1}^{d^{\prime}} A_{i}^{2}
$$

is the sublaplacian and

$$
H_{1}=\sum_{i=1}^{d^{\prime}} c_{i} A_{i}+c_{0} I
$$


Now it can be deduced from the estimates of [22, Corollary IV.4.19] that $H_{1}$ is a small perturbation of $H_{0}$ and that the closure of $H$ generates a continuous semigroup. Alternatively, it follows from Statement I of Proposition 4.3 that the closure generates a continuous holomorphic semigroup. The proof is as follows.

First, Statement I of the proposition implies the existence of a $c>0$ such that

$$
\left\|H_{1} x\right\| \leq \varepsilon\left\|\bar{H}_{0} x\right\|+c \varepsilon^{-1}\|x\|
$$

for all $x \in D\left(\bar{H}_{0}\right)$ and $\varepsilon \in\langle 0,1]$. Secondly, $\bar{H}_{0}$ generates a continuous holomorphic semigroup $S^{(0)}$. Now if the representation is strongly continuous $S^{(0)}$ is also strongly continuous and it follows immediately from the perturbation theory of holomorphic semigroups and (20) that the closure of $H$ generates a strongly continuous holomorphic semigroup $S$ with the same holomorphy sector as $S^{(0)}$. If, however, the representation is weakly* continuous the proof is somewhat more circuitous. Then (20) gives

$$
\left\|H_{1} S_{t}^{(0)} x\right\| \leq \varepsilon\left\|\bar{H}_{0} S_{t}^{(0)} x\right\|+c \varepsilon^{-1}\left\|S_{t}^{(0)} x\right\| .
$$

Consequently, the continuity bounds $\left\|S_{t}^{(0)}\right\| \leq M e^{\omega t}$, the holomorphy bounds $\left\|\bar{H}_{0} S_{t}^{(0)}\right\|$ $\leq M t^{-1} e^{\omega t}$, the choice $\varepsilon=t^{1 / 2}$ and the semigroup property lead to bounds

$$
\left\|\bar{H}_{1} S_{t}^{(0)}\right\| \leq M^{\prime} t^{-1 / 2} e^{\omega^{\prime} t}
$$

for all $t>0$. Therefore the weak*-weak*-closure $\bar{H}$ of $H$ generates a weakly* continuous semigroup $S$ by 'time-dependent' perturbation theory (see, for example, [3, Theorem 3.1.33]). Moreover, $S$ is related to $S^{(0)}$ by the perturbation series

$$
S_{t}=\sum_{n \geq 0} S_{t}^{(n)}
$$

where the terms of the series are defined inductively by the relation

$$
S_{t}^{(n)}=-\int_{0}^{t} d s S_{t-s}^{(0)} \bar{H}_{1} S_{s}^{(n-1)} .
$$

The perturbation series is norm-convergent for small values of $t$, because of the estimates (21). It then remains to deduce that $S$ is holomorphic. But this can be established by a duality argument.

Let $\mathscr{F}$ denote the dual of $\mathscr{X}$ and $\mathscr{F}_{n}^{\prime}$ the $C^{n}$-subspaces analogous to $\mathscr{X}_{n}^{\prime}$ but associated with the strongly continuous dual representation $U_{*}$ of $G$ on $\mathscr{F}$. If $H^{\dagger}$ denotes the formal adjoint of $H$, that is, the restriction of the adjoint $H^{*}$ to $\mathscr{F}_{2}^{\prime}$, then $H^{\dagger}$ is a second-order subelliptic operator affiliated with the dual representation of the form $H^{\dagger}=H_{0}+H_{1}^{\prime}$ where $H_{0}$ is again the sublaplacian and $H_{1}^{\prime}$ is a first-order 
operator. Now Statement I of Proposition 4.3 applied to the dual representation gives bounds

$$
\left\|H_{1}^{\prime} f\right\| \leq \varepsilon\left\|\bar{H}_{0} f\right\|+c \varepsilon^{-1}\|f\|
$$

for all $f \in D\left(\bar{H}_{0}\right)$ and $\varepsilon \in\langle 0,1]$. Hence the foregoing argument applied to $H^{\dagger}$ establishes that the closure of the operator generates a strongly continuous holomorphic semigroup $T$ on $\mathscr{F}$. Then $T^{*}$ is a weakly* continuous holomorphic semigroup on $\mathscr{X}$ with generator $\left(H^{\dagger}\right)^{*}$ and the same holomorphy sector as $T$. But $\left(H^{\dagger}\right)^{*}$ extends $\bar{H}$ which is itself a generator. Since a semigroup generator cannot have a strict generator extension it follows that $\left(H^{\dagger}\right)^{*}=\bar{H}$ and $S=T$. Thus $S$ is a holomorphic semigroup.

Next we turn to the extension of the results of the previous sections with the operator $H$ replacing the sublaplacian and the semigroup $S$ replacing the semigroup generated by the sublaplacian. It might seem that this extension to the perturbed semigroup must involve complex perturbation calculations but these can be circumvented by use of interpolation theory. Let $n \in \mathbb{N}$ and $\gamma \in\langle 0, n\rangle$. Then by Theorem 3.1 one deduces that

$$
\mathscr{X}_{\gamma}^{n, S^{(0)}}=\left(\mathscr{X}, D\left(\bar{H}_{0}^{n}\right)\right)_{\gamma / n, p ; \mathrm{K}}
$$

and

$$
\mathscr{X}_{\gamma}^{n, S}=\left(\mathscr{X}, D\left(\bar{H}^{n}\right)\right)_{\gamma / n, p ; \mathrm{K}} .
$$

Therefore if $D\left(\bar{H}_{0}^{n}\right)=D\left(\bar{H}^{n}\right)$ then

$$
\mathscr{X}_{\gamma / 2}^{n, S}=\mathscr{X}_{\gamma / 2}^{n, S^{(0)}}=\left(\mathscr{X}, \mathscr{X}_{n}^{\prime}\right)_{\gamma}
$$

and the corresponding norms are equivalent. This is the general tactic for the proof of the subsequent theorem but it is necessary to exercise some care in appealing to interpolation theory for weakly* continuous semigroups because the theory is usually formulated for strongly continuous groups.

THEOREM 5.1. Let

$$
H=-\sum_{i, j=1}^{d^{\prime}} c_{i j} A_{i} A_{j}+\sum_{i=1}^{d^{\prime}} c_{i} A_{i}+c_{0} I
$$

where $C=\left(c_{i j}\right)$ is a real-valued strictly positive-definite matrix and $c_{0}, c_{1}, \ldots, c_{d^{\prime}} \in$ $\mathbb{C}$. Further let $S$ be the continuous holomorphic semigroup generated by the closure $\bar{H}$ of $H$. Then one has the following.

I. If $n>\gamma>0$ then $\left(\mathscr{X}, \mathscr{X}_{n}^{\prime}\right)_{\gamma}=\mathscr{X}_{\gamma / 2}^{n, S}$ and the norms $\|\cdot\|_{\gamma}^{(0, n)}$ and $\|\cdot\|_{\gamma / 2}^{n, S}$ are equivalent.

II. $\quad S_{t}$ maps $\mathscr{X}$ into $\mathscr{X}_{\infty}$ for all $t>0$. 
III. The conclusions of Theorem 4.1 are valid with the operator $H$ replacing the sublaplacian.

IV. The inequalities of Propositions 4.3 and 4.4 are valid if in addition we define the spaces $D\left(\bar{H}^{\gamma / 2}\right)$ to be Banach spaces of order $\gamma$ if $S$ is uniformly bounded.

V. If $p \in[1, \infty)$ and $n>\gamma>0$ then the restriction of $S$ to $\left(\mathscr{X}, \mathscr{X}_{n}^{\prime}\right)_{\gamma}$ is a strongly continuous holomorphic semigroup.

ProOF. First we prove that $S_{t}$ maps $\mathscr{X}_{\infty}$ into $\mathscr{X}_{\infty}$. Let $\tilde{X}$ be the subspace on which $U$ acts strongly continuous and let $\widetilde{U}$ be the restriction of $U$ to $\widetilde{X}$. Let $n \in \mathbb{N}$ and let $\widetilde{U}_{n}$ denote the restriction of $\widetilde{U}$ to the Banach space $\widetilde{\mathscr{X}}_{n}$ of $C^{n}$-vectors with respect to the full vector space basis $a_{1}, \ldots, a_{d^{\prime}}, \ldots, a_{d}$. Now one can repeat the perturbation arguments given above with the strongly continuous representation $\left(\widetilde{\mathscr{X}}_{n}, G, \widetilde{U}_{n}\right)$ replacing the representation $(\mathscr{X}, G, U)$. Therefore if

$$
K=-\sum_{i, j=1}^{d^{\prime}} c_{i j} A_{i} A_{j}+\sum_{i=1}^{d^{\prime}} c_{i} A_{i}+c_{0} I
$$

is the operator in $\widetilde{\mathscr{X}}_{n}$ with domain $\left(\widetilde{\mathscr{X}}_{n}\right)_{2}^{\prime}$ then the closure $\bar{K}$ of $K$ in $\widetilde{\mathscr{X}}_{n}$ generates a holomorphic semigroup $T$ on $\widetilde{\mathscr{X}}_{n}$. But if $x \in \widetilde{\mathscr{X}}_{n}$ one has

$$
S_{t} x-T_{t} x=\int_{0}^{t} d s S_{t-s}(\bar{K}-\bar{H}) T_{s} x=0 .
$$

It follows that $T_{t} \subseteq S_{t}$ for all $t \geq 0$. Hence

$$
S_{t} \mathscr{X}_{\infty} \subseteq S_{t} \widetilde{\mathscr{X}}_{n}=T_{t} \widetilde{\mathscr{X}}_{n} \subseteq \widetilde{\mathscr{X}}_{n} \subseteq \mathscr{X}_{n}
$$

and therefore

$$
S_{t} \mathscr{X}_{\infty} \subseteq \bigcap_{n=1}^{\infty} \mathscr{X}_{n}=\mathscr{X}_{\infty}
$$

Secondly, assume that the representation, and hence the semigroup $S$, is strongly continuous. Since $S_{t}$ leaves $\mathscr{X}_{\infty}$ invariant we can apply [19, Corollary 1.3] and it follows that $\mathscr{X}_{\infty}$ is dense in $D\left(\bar{H}^{n}\right)$ for all $n \in \mathbb{N}$. Next, by the discussion at the beginning of the section, we may assume that $H$ has the form $H=H_{0}+H_{1}$ where $H_{0}$ is the sublaplacian and $H_{1}$ is a first-order operator. If $S^{(0)}$ is the semigroup generated by $\bar{H}_{0}$ and $n \in \mathbb{N}$ then there exists an operator $P$ of order $2 n-1$ in the operators $A_{1}, \ldots, A_{d^{\prime}}$ such that

$$
H^{n} x=H_{0}^{n} x+P x
$$

for all $x \in \mathscr{X}_{\infty}$. By Proposition 4.3.I one has bounds

$$
\|P x\| \leq c_{1}\|x\|_{2 n-1}^{\prime} \leq\left\|\bar{H}_{0}^{n} x\right\| / 2+c_{2}\|x\|
$$


valid for all $x \in \mathscr{X}_{\infty}$. Consequently

$$
\left\|\bar{H}_{0}^{n} x\right\| \leq\left\|\bar{H}^{n} x\right\|+\|P x\| \leq\left\|\bar{H}^{n} x\right\|+\left\|\bar{H}_{0}^{n} x\right\| / 2+c_{2}\|x\|
$$

and

$$
\left\|\bar{H}_{0}^{n} x\right\| \leq 2\left\|\bar{H}^{n} x\right\|+2 c_{2}\|x\|
$$

for all $x \in \mathscr{X}_{\infty}$. Now since $\mathscr{X}_{\infty}$ is dense in $D\left(\bar{H}^{n}\right)$ and $D\left(\bar{H}_{0}^{n}\right)$ is complete it follows that $D\left(\bar{H}^{n}\right)$ is continuously embedded in $D\left(\bar{H}_{0}^{n}\right)$. By a similar, but easier, argument the opposite inclusion is also valid. Therefore

$$
D\left(\bar{H}_{0}^{n}\right)=D\left(\bar{H}^{n}\right)
$$

and the norms $x \mapsto\left\|\bar{H}_{0}^{n} x\right\|+\|x\|$ and $x \mapsto\left\|\bar{H}^{n} x\right\|+\|x\|$ are equivalent. Statement I now follows by the argument based on interpolation theory given before the statement of the theorem.

Next suppose that the representation, and hence the semigroup $S$, is weakly* continuous. It follows from the perturbation series for $S$ and the estimate (21) that

$$
\left\|S_{t} x-x\right\| \leq\left\|S_{t}^{(0)} x-x\right\|+c t^{1 / 2}\|x\|
$$

and

$$
\left\|S_{t}^{(0)} x-x\right\| \leq\left\|S_{t} x-x\right\|+c t^{1 / 2}\|x\|
$$

for a suitable $c>0$ and all small $t>0$. Therefore the strong continuity subspaces of $S$ and $S^{(0)}$ coincide. But the strong continuity subspace for $S^{(0)}$ is $\widetilde{\mathscr{X}}$ by the proof of Corollary 3.4. Further let $\widetilde{S}$ and $\widetilde{S}^{(0)}$ denote the strongly continuous restrictions of the semigroups $S$ and $S^{(0)}$ to $\widetilde{\mathscr{X}}$. By Theorem 3.2 and Corollary 3.4

$$
\left(\mathscr{X}, \mathscr{X}_{n}^{\prime}\right)_{\gamma}=\left(\widetilde{X}, \widetilde{\mathscr{X}}_{n}^{\prime}\right)_{\gamma}=\widetilde{\mathscr{X}}_{\gamma / 2}^{n, \tilde{S}^{(0)}} \text {. }
$$

By Theorem 3.1 also $\mathscr{X}_{\gamma}^{n, S^{(0)}}=\widetilde{\mathscr{X}}_{\gamma}^{n, \tilde{S}^{(0)}}$. Therefore Statement I follows once we establish that $\widetilde{\mathscr{X}}_{\gamma}^{n, \tilde{S}}=\widetilde{\mathscr{X}}_{\gamma}^{n, \widetilde{S}^{(0)}}$. But this is a problem in the strongly continuous case and which has already been solved. Hence the proof of Statement I is complete in the weakly* continuous case.

If the representation is strongly continuous then Statement II follows from the holomorphy of $S$ and equality (22) because

$$
S_{t} \mathscr{X} \subseteq \bigcap_{n=1}^{\infty} D\left(\bar{H}^{n}\right)=\bigcap_{n=1}^{\infty} D\left(\bar{H}_{0}^{n}\right)=\mathscr{X}_{\infty} .
$$

Alternatively, if the representation is weakly* continuous $S_{t / 2} \mathscr{X} \subseteq \widetilde{\mathscr{X}}$ for all $t>0$ by holomorphy. Then, however,

$$
S_{t} \mathscr{X} \subseteq S_{t / 2} \widetilde{X}=\tilde{S}_{t / 2} \widetilde{X} \subseteq \widetilde{X}_{\infty} \subseteq \mathscr{X}_{\infty}
$$


for all $t>0$ where we have used the previous result for the strongly continuous representation $(\widetilde{\mathscr{X}}, G, \widetilde{U})$.

The proofs of Statements III and IV are precisely the same as the proofs for $H_{0}$ and $S^{(0)}$, but now use Statement I instead of Theorem 3.2. The type of continuity plays no significant role.

Finally, the proof that for $p<\infty$ the restriction of $S$ to the Lipschitz space $\left(\mathscr{X}, \mathscr{X}_{n}^{\prime}\right)_{\gamma}$ is a strongly continuous semigroup is the same as the proof given for $S^{(0)}$ just prior to Corollary 3.4. Then by Statements III and IV one obtains bounds

$$
\left\|\bar{H} S_{t} x\right\|_{\gamma}^{(0, n)} \leq c\left\|S_{t} x\right\|_{\gamma ; 2}^{(0, n)} \leq C t^{-1}\|x\|_{\gamma}^{(0, n)}
$$

valid for all $t \in\langle 0,1]$ and $x \in \mathscr{X}$. Therefore $S$ is a holomorphic semigroup on $\left(\mathscr{X}, \mathscr{X}_{n}^{\prime}\right)_{\gamma}$.

The semigroups generated by subelliptic operators with real coefficients have integral kernels which satisfy Gaussian bounds (see [22, Chapter IV]). The same is true in the current context of operators with complex first-order coefficients. This can be established by a simple variation of the proofs for operators with real coefficients. Specifically one can repeat the reasoning of [22, Chapter IV, Sections 2 and 4]. One estimates various properties of the semigroup $S$ associated with the subelliptic operator $H$ and left translations on the function spaces $L_{p}(G ; d g)$ and $L_{p}(G ; d \hat{g})$, where $d g$ and $d \hat{g}$ denote left and right invariant Haar measure respectively. It is important for these calculations that $S_{t} \mathscr{X} \subseteq \mathscr{X}_{\infty}$ but this has already been established. The crucial features of the calculations are quadratic form estimates and for this it is important that on $L_{2}(G ; d g)$ one has

$$
\begin{aligned}
\left|\left(\varphi, H_{1} \varphi\right)\right| & \leq\|\varphi\|_{2}|c|\left(\sum_{i=1}^{d^{\prime}}\left\|A_{i} \varphi\right\|_{2}^{2}\right)^{1 / 2} \\
& \leq|c|\left(\varepsilon \sum_{i=1}^{d^{\prime}}\left\|A_{i} \varphi\right\|_{2}^{2}+(1 /(4 \varepsilon))\|\varphi\|_{2}^{2}\right) .
\end{aligned}
$$

First one deduces that $S_{t}$ is bounded as an operator from $L_{1}(G ; d \hat{g})$ to $L_{\infty}$, for each $t>0$. But then by the Dunford-Pettis theorem $S$ is determined by an integral kernel $K$. Secondly, one derives a set of Gaussian bounds on $K$, and its derivatives with respect to $t$, of the form

$$
\left|\partial_{t}^{n} K_{t}(g)\right| \leq a_{n} t^{-n-D^{\prime} / 2} e^{\omega t} e^{-b\left(|g|^{\prime}\right)^{2} / t}
$$

for all $g \in G, t>0$, and $n \in \mathbb{N}_{0}$. If $G$ is unimodular, or polynomial, these bounds can be improved exactly as in the case of real coefficients. Moreover, the analyticity of $K$ jointly in $t$ and $g$ follows by the reasoning for real coefficients. But for complex 
coefficients the kernel is not positive and hence one cannot hope to obtain lower bounds. Finally we note that one can also obtain bounds on the left derivatives of $K$ in the directions $a_{1}, \ldots, a_{d^{\prime}}$ by the methods used for real coefficients. These bounds take the form

$$
\left|A_{i} K_{t}(g)\right| \leq a t^{-1 / 2-D^{\prime} / 2} e^{\omega t} e^{-b\left(|g|^{\prime}\right)^{2} / t}
$$

for all $g \in G, t>0$, and $i \in\left\{1, \ldots, d^{\prime}\right\}$.

\section{The domain of $\bar{H}^{N}$}

The Lipschitz spaces can be used to obtain estimates for the domain of the closure of the operator

$$
H=-\sum_{i, j=1}^{d^{\prime}} c_{i j} A_{i} A_{j}+\sum_{i=1}^{d^{\prime}} c_{i} A_{i}+c_{0} I
$$

and its powers. In general the estimates that we obtain in this section are optimal but in particular cases they can be improved. This will be demonstrated for unitary representations in the next section.

If $a_{0}, a_{1}, \ldots, a_{d^{\prime}}$ is an algebraic basis of the Lie algebra $\mathfrak{g}$,

$$
H=-\sum_{i=1}^{d^{\prime}} A_{i}^{2}+A_{0}+c I,
$$

and the representation is strongly continuous, then Jørgensen, [11, Theorem 2.1 and Corollary 2.1], proved that for all $n \in \mathbb{N}$ there exists an $N \in \mathbb{N}$ such that $D\left(\bar{H}^{N}\right) \subseteq \mathscr{X}_{n}$ and

$$
\|x\|_{n} \leq c\left(\left\|\bar{H}^{N} x\right\|+\|x\|\right)
$$

for all $x \in D\left(\bar{H}^{N}\right)$ and a suitable $c>0$. In his proof the existence of the $N$ follows from the closed graph theorem and it is not clear how it depends on $n$. Alternatively, if $a_{1}, \ldots, a_{d^{\prime}}$ is a full vector space basis for $\mathfrak{g}$ then it follows from [22, Theorem II.5.5] that

$$
D\left(\bar{H}^{N}\right) \subseteq\left(\mathscr{X}, \mathscr{X}_{2 N}\right)_{2 N-\varepsilon} \subseteq \mathscr{X}_{2 N-1}
$$

for all $\varepsilon \in\langle 0,1\rangle$ and the inclusions are continuous. In this section we give inclusions of this kind for operators of the form (23). The inclusions are valid for both kinds of continuity, strong or weak*.

First we compare the Lipschitz spaces which interpolate between the representation space $\mathscr{X}$ and the subelliptic $C^{n}$-subspaces $\mathscr{X}_{n}^{\prime}$ with the Lipschitz spaces which interpolate between $\mathscr{X}$ and the full $C^{n}$-subspaces $\mathscr{X}_{n}$. Since we are dealing with two kinds of Lipschitz spaces in this section, $\left(\mathscr{X}_{n_{1}}, \mathscr{X}_{n_{2}}\right)_{\gamma}$ and $\left(\mathscr{X}_{n_{1}}^{\prime}, \mathscr{X}_{n_{2}}^{\prime}\right)_{\gamma}$, we denote 
the norm on $\left(\mathscr{X}_{n_{1}}^{\prime}, \mathscr{X}_{n_{2}}^{\prime}\right)_{\gamma}$ by $\|\cdot\|_{\gamma}^{\left(n_{1}^{\prime}, n_{2}^{\prime}\right)}$ and the norm on $\left(\mathscr{X}_{n_{1}}, \mathscr{X}_{n_{2}}\right)_{\gamma}$ by $\|\cdot\|_{\gamma}^{\left(n_{1}, n_{2}\right)}$. Also we denote the space

$$
\bigcap_{k=0}^{n} \bigcap_{i_{1}, \ldots, i_{k} \in\left\{1, \ldots, d^{\prime}\right\}}\left\{x \in D\left(A_{i_{1}} \ldots A_{i_{k}}\right): A_{i_{1}} \ldots A_{i_{k}} x \in\left(\mathscr{X}_{n_{1}}^{\prime}, \mathscr{X}_{n_{2}}^{\prime}\right)_{\gamma}\right\}
$$

by $\left(\mathscr{X}_{n_{1}}^{\prime}, \mathscr{X}_{n_{2}}^{\prime}\right)_{\gamma ; n}$ and we use $\left(\mathscr{X}_{n_{1}}, \mathscr{X}_{n_{2}}\right)_{\gamma ; n}$ to denote the space

$$
\bigcap_{k=0}^{n} \bigcap_{i_{1}, \ldots, i_{k} \in\{1, \ldots, d\}}\left\{x \in D\left(A_{i_{1}} \ldots A_{i_{k}}\right): A_{i_{1}} \ldots A_{i_{k}} x \in\left(\mathscr{X}_{n_{1}}, \mathscr{X}_{n_{2}}\right)_{\gamma}\right\}
$$

The basic comparison result is given by the following lemma.

LEMMA 6.1. If $r$ denotes the rank of the algebraic basis $a_{1}, \ldots, a_{d^{\prime}}$ and $n>\gamma r>0$ then

$$
\left(\mathscr{X}, \mathscr{X}_{n}^{\prime}\right)_{\gamma r} \subseteq\left(\mathscr{X}, \mathscr{X}_{n}\right)_{\gamma} \subseteq\left(\mathscr{X}, \mathscr{X}_{n}^{\prime}\right)_{\gamma}
$$

and the embeddings are continuous.

PROOF. By Theorem 2.1 we have the following equalities.

$$
\begin{aligned}
\left(\mathscr{X}, \mathscr{X}_{n}^{\prime}\right)_{\gamma r} & =\left(\mathscr{X}, \mathscr{X}_{n r}^{\prime}\right)_{\gamma r}=\left(\mathscr{X}, \mathscr{X}_{n r}^{\prime}\right)_{\gamma / n, p ; \mathrm{K}} \\
\left(\mathscr{X}, \mathscr{X}_{n}\right)_{\gamma} & =\left(\mathscr{X}, \mathscr{X}_{n}\right)_{\gamma / n, p ; \mathrm{K}} \\
\left(\mathscr{X}, \mathscr{X}_{n}^{\prime}\right)_{\gamma} & =\left(\mathscr{X}, \mathscr{X}_{n}^{\prime}\right)_{\gamma / n, p ; \mathrm{K}} .
\end{aligned}
$$

But $\mathscr{X}_{n r}^{\prime} \subseteq \mathscr{X}_{n} \subseteq \mathscr{X}_{n}^{\prime}$ and the inclusions are continuous. So by interpolation theory, [6, Theorem 3.2.23], it follows that

$$
\left(\mathscr{X}, \mathscr{X}_{n r}^{\prime}\right)_{\gamma / n, p ; \mathrm{K}} \subseteq\left(\mathscr{X}, \mathscr{X}_{n}\right)_{\gamma / n, p ; \mathrm{K}} \subseteq\left(\mathscr{X}, \mathscr{X}_{n}^{\prime}\right)_{\gamma / n, p ; \mathrm{K}}
$$

and the inclusions are continuous.

REMARK. Another proof of this lemma can be obtained from the characterization $\left(\mathscr{X}, \mathscr{X}_{n}^{\prime}\right)_{\gamma}=\mathscr{X}_{\gamma}^{n, \omega}$, and a similar equality for the Lipschitz space $\left(\mathscr{X}, \mathscr{X}_{n}\right)_{\gamma}$, by using the inequalities

$$
c_{1}|g| \leq|g|^{\prime} \leq c_{2}|g|^{1 / r}
$$

valid for all $g \in G$ with $|g|^{\prime} \leq 1$ (see [14, Proposition 1.1]).

The embeddings of the lemma allow one to translate regularity properties with respect to the algebraic basis into regularity properties with respect to the full basis. 
THEOREM 6.2. Let

$$
H=-\sum_{i, j=1}^{d^{\prime}} c_{i j} A_{i} A_{j}+\sum_{i=1}^{d^{\prime}} c_{i} A_{i}+c_{0}
$$

where $C=\left(c_{i j}\right)$ is a real-valued strictly positive-definite matrix and $c_{0}, c_{1}, \ldots, c_{d^{\prime}} \in$ C. Let $r$ be the rank of the algebraic basis $a_{1}, \ldots, a_{d^{\prime}}$.

I. If $n, N \in \mathbb{N}$ satisfy $n<2 N / r$ then $D\left(\bar{H}^{N}\right) \subseteq \mathscr{X}_{n}$. Moreover, if the semigroup generated by the closure $\bar{H}$ of $H$ is uniformly bounded and $\alpha>0$ then

$$
D\left(\bar{H}^{\alpha}\right) \subseteq\left(\mathscr{X}_{k}, \mathscr{X}_{k+1}\right)_{\gamma}=\left(\mathscr{X}, \mathscr{X}_{1}\right)_{\gamma ; k}
$$

where $k$ is the largest integer strictly smaller than $2 \alpha / r$ and $\gamma \in\langle 0,2 \alpha / r-k\rangle$. Both sets of embeddings are continuous.

II. Suppose the semigroup generated by the closure $\bar{H}$ of $H$ is uniformly bounded. If $p=\infty$ and $\alpha>0$ then

$$
D\left(\bar{H}^{\alpha}\right) \subseteq\left(\mathscr{X}_{k}, \mathscr{X}_{k+2}\right)_{\gamma}=\left(\mathscr{X}, \mathscr{X}_{2}\right)_{\gamma ; k}
$$

where $k$ is the largest integer strictly smaller than $2 \alpha / r$ and $\gamma=2 \alpha / r-k$. The embeddings are again continuous.

III. Suppose the semigroup generated by the closure $\bar{H}$ of $H$ is uniformly bounded. If $\gamma>0, \alpha>0, n>\gamma+2 \alpha / r$ and $x \in D\left(\bar{H}^{\alpha}\right)$ then $\bar{H}^{\alpha} x \in$ $\left(\mathscr{X}, \mathscr{X}_{n}\right)_{\gamma}$ implies $x \in\left(\mathscr{X}, \mathscr{X}_{n}\right)_{\gamma+2 \alpha / r}$. Moreover, there exists a $c>0$, depending on $n, \gamma$ and $\alpha$, such that

$$
\|x\|_{\gamma+2 \alpha / r}^{(0, n)} \leq c\left(\left\|\bar{H}^{\alpha} x\right\|_{\gamma}^{(0, n)}+\|x\|_{\gamma}^{(0, n)}\right)
$$

for all $x \in D\left(H^{\alpha}\right)$.

PROOF. For Statement I we only need to prove the second part because the first part follows from Theorem 2.1.I.

Let $\varepsilon \in\langle 0,2 \alpha\rangle$. Then by Theorem 5.1.IV it follows that there exists a $c>0$ such that

$$
\|x\|_{2 \alpha-\varepsilon}^{\left(\sigma, m^{\prime}\right)} \leq c\left(\left\|\bar{H}^{\alpha} x\right\|+\|x\|\right)
$$

for all $x \in D\left(\bar{H}^{\alpha}\right)$. In particular $D\left(\bar{H}^{\alpha}\right) \subseteq\left(\mathscr{X}, \mathscr{X}_{m}^{\prime}\right)_{2 \alpha-\varepsilon}$. Now the second part of Statement I follows from the previous lemma and Theorem 2.1.

If $p=\infty$ it follows from Theorem 5.1.IV that

$$
D\left(\bar{H}^{(\gamma+k) r / 2}\right) \subseteq\left(\mathscr{X}, \mathscr{X}_{n}^{\prime}\right)_{(\gamma+k) r} \subseteq\left(\mathscr{X}, \mathscr{X}_{n}\right)_{\gamma+k}
$$


for $n>\gamma+k$ and the desired inclusion is established. This proves Statement II.

Finally we prove Statement III. First note that Statement II shows that the operator $(I+\bar{H})^{-\alpha}$ is a bounded operator from $\mathscr{X}$ into $\left(\mathscr{X}, \mathscr{X}_{n}\right)_{2 \alpha / r}$ where the Lipschitz space is with respect to $p=\infty$. Secondly, let $N \in \mathbb{N}, N>\gamma$. If $(\widetilde{\mathscr{X}}, G, \widetilde{U})$ denotes the strongly continuous component of the representation and $\left(\widetilde{\mathscr{X}}_{N}, G, \widetilde{U}_{N}\right.$ ) the representation obtained by restricting $\widetilde{U}$ to the $C^{N}$-subspace $\widetilde{\mathscr{X}}_{N}$, then by replacing $(\mathscr{X}, G, U)$ by $\left(\widetilde{\mathscr{X}}_{N}, G, \widetilde{U}_{N}\right)$ in the previous argument one deduces that $(I+\bar{H})^{-\alpha}$ is a bounded operator from $\widetilde{\mathscr{X}}_{N}$ into $\left(\widetilde{\mathscr{X}}_{N}, \widetilde{\mathscr{X}}_{N+n}\right)_{2 \alpha / r}$ where the Lipschitz space is still with respect to $p=\infty$ and we have assumed that $n>N+2 \alpha / r$. But $\left(\widetilde{\mathscr{X}}_{N}, \widetilde{\mathscr{X}}_{N+n}\right)_{2 \alpha / r}=\left(\mathscr{X}_{N}, \mathscr{X}_{N+n}\right)_{2 \alpha / r}$ by Corollary 3.4. Moreover $\left(\mathscr{X}_{N}, \mathscr{X}_{N+n}\right)_{2 \alpha / r}=\left(\mathscr{X}, \mathscr{X}_{n}\right)_{N+2 \alpha / r}$ by Theorem 2.1 . Therefore

$$
(I+\bar{H})^{-\alpha}: \widetilde{\mathscr{X}} \rightarrow\left(\mathscr{X}, \mathscr{X}_{n}\right)_{2 \alpha /(n r), \infty ; \mathrm{K}}
$$

and

$$
(I+\bar{H})^{-\alpha}: \widetilde{\mathscr{X}}_{N} \rightarrow\left(\mathscr{X}, \mathscr{X}_{n}\right)_{N / n+2 \alpha /(n r), \infty ; \mathrm{K}}
$$

are bounded operators. So by interpolation, [6, Theorem 3.2.23], and another application of Corollary 3.4 one deduces that $(I+\bar{H})^{-\alpha}:\left(\mathscr{X}, \mathscr{X}_{N}\right)_{\gamma / N, p ; \mathrm{K}} \rightarrow\left(\left(\mathscr{X}, \mathscr{X}_{n}\right)_{2 \alpha /(n r), \infty ; \mathrm{K}},\left(\mathscr{X}, \mathscr{X}_{n}\right)_{N / n+2 \alpha /(n r), \infty ; \mathrm{K}}\right)_{\gamma / N, p ; \mathrm{K}}$ is a bounded operator for all $p \in[1, \infty]$. Thirdly, by the reiteration theorem, $[6$, Theorem 3.2.20], together with [6, Corollary 3.2.17]

$$
\left(\left(\mathscr{X}, \mathscr{X}_{n}\right)_{2 \alpha /(n r), \infty ; \mathrm{K}},\left(\mathscr{X}, \mathscr{X}_{n}\right)_{N / n+2 \alpha /(n r), \infty ; \mathrm{K}}\right)_{\gamma / N, p ; \mathrm{K}}=\left(\mathscr{X}, \mathscr{X}_{n}\right)_{\gamma / n+2 \alpha /(n r), p ; \mathrm{K}}
$$

and the norms are equivalent. So

$$
(I+\bar{H})^{-\alpha}:\left(\mathscr{X}, \mathscr{X}_{n}\right)_{\gamma} \rightarrow\left(\mathscr{X}, \mathscr{X}_{n}\right)_{\gamma+2 \alpha / r}
$$

is bounded for all $p \in[1, \infty]$. In particular, there exists a $c>0$ such that for all $x \in\left(\mathscr{X}, \mathscr{X}_{n}\right)_{\gamma}$

$$
\left\|(I+\bar{H})^{-\alpha} x\right\|_{\gamma+2 \alpha / r}^{(0, n)} \leq c\|x\|_{\gamma}^{(0, n)}
$$

Therefore,

$$
\|x\|_{\gamma+2 \alpha / r}^{(0, n)} \leq c\left\|(I+\bar{H})^{\alpha} x\right\|_{\gamma}^{(0, n)}
$$

for all $x \in D\left((I+\bar{H})^{\alpha}\right)$ such that $(I+\bar{H})^{\alpha} x \in\left(\mathscr{X}, \mathscr{X}_{n}\right)_{\gamma}$. Fourthly, by [22, Lemma II.3.2], there exists a bounded operator $B$ on $\mathscr{X}$ such that

$$
(I+\bar{H})^{\alpha} x=\bar{H}^{\alpha} x+B x
$$


for all $x \in D\left(\bar{H}^{\alpha}\right)=D\left((I+\bar{H})^{\alpha}\right)$. By the above reasoning $B$ is also bounded on $\tilde{\mathscr{X}}_{N}$ and then by interpolation it is bounded on $\left(\mathscr{X}, \mathscr{X}_{n}\right)_{\gamma}$. Thus if $x \in D\left(\bar{H}^{\alpha}\right)=$ $D\left((I+\bar{H})^{\alpha}\right)$ one has $(I+\bar{H})^{\alpha} x \in\left(\mathscr{X}, \mathscr{X}_{n}\right)_{\gamma}$ if, and only if, $\bar{H}^{\alpha} x \in\left(\mathscr{X}, \mathscr{X}_{n}\right)_{\gamma}$ and

$$
\|x\|_{\gamma+2 \alpha / r}^{(0, n)} \leq c^{\prime}\left(\left\|\bar{H}^{\alpha} x\right\|_{\gamma}^{(0, n)}+\|x\|_{\gamma}^{(0, n)}\right)
$$

for some $c^{\prime}>0$, independent of $x$. This proves Statement III.

Application of Theorem 2.1 with a full vector space basis of gives the identifications

$$
\left(\mathscr{X}_{k}, \mathscr{X}_{k+1}\right)_{\gamma}=\left(\mathscr{X}, \mathscr{X}_{k+1}\right)_{\gamma+k}=\left(\mathscr{X}, \mathscr{X}_{1}\right)_{\gamma ; k}
$$

where the derivatives are in all possible directions. Therefore the second part of the first statement of the theorem can be reformulated as a general differentiability statement. Each $x \in D\left(\bar{H}^{\alpha}\right)$ is a $k$-times differentiable element and the $k$-fold derivatives are in the Lipschitz space $\left(\mathscr{X}, \mathscr{X}_{1}\right)_{\gamma}$. In this form the theorem is an extension of the usual elliptic regularity theorem for elliptic differential operators. This analogy indicates that the statement of the theorem is probably optimal unless one makes more specific assumptions on the form of the representation or the differential operator. This is confirmed by the example at the end of the next section.

The conclusions of Theorem 6.2 can be directly compared to the regularity results of Rothschild and Stein [23] if these latter are restricted to the Lie group setting. Rothschild and Stein deal with subelliptic operators acting on various function spaces over a manifold $M$. In particular if $M=G$ then their results apply to the $L_{p}$-spaces $L_{p}(G ; d g)$, constructed with respect to left-invariant Haar measure $d g$, and to various related interpolation spaces. The corresponding subelliptic operators are constructed from the representation of $G$ by left, or right, translations on these spaces. Theorem 16 of [23] encapsulates the nature of their regularity results. It has four parts the second and third of which are directly comparable with the above theorem. Part (b) of [23, Theorem 16] implies that $(\lambda I+\bar{H})^{-1}$ is bounded as an operator from the interpolation space $\left(L_{\infty}, L_{\infty ; n}\right)_{\gamma / n, \infty ; K}$ to the interpolation space $\left(L_{\infty}, L_{\infty ; n}\right)_{(\gamma+2 / r) / n, \infty ; K}$ for all large $\lambda$, where $L_{\infty ; n}$ denotes the $C^{n}$-subspace of $L_{\infty}$ with respect to left translations. Theorem 6.2 establishes, however, that $(\lambda I+\bar{H})^{-1}$ is bounded as an operator from $\left(L_{\infty}, L_{\infty ; n}\right)_{\gamma / n, p ; K}$ to $\left(L_{\infty}, L_{\infty ; n}\right)_{(\gamma+2 / r) / n, p ; K}$ for all $p \in[1, \infty]$, that is, it extends the Rothschild-Stein result to a broader class of spaces. Similarly [23, Theorem 16 Part (c)] states that $(\lambda I+\bar{H})^{-1}$ is bounded as an operator from $L_{\infty}$ to $\left(L_{\infty}, L_{\infty ; n}\right)_{2 /(n r), \infty ; K}$ but Theorem 6.2 establishes that it is in fact bounded from $L_{p}$ to $\left(L_{p}, L_{p ; n}\right)_{2 /(n r), \infty ; K}$ for all $p \in[1, \infty]$. Further comparison with the Rothschild-Stein results is given at the end of the next section. 


\section{Unitary representations}

Although the regularity statements of Theorem 6.2 are in general optimal they can be improved in special cases. Next we demonstrate that this is indeed true for unitary representations and we establish among other things the subelliptic equivalent of the results of Nelson referred to in the introduction. The proof again utilizes the Lipschitz spaces. In the Hilbert space context these spaces have a particularly simple form for the special value $p=2$ and this will be exploited throughout the section.

We begin with the appropriate characterization of the Lipschitz spaces.

LEMMA 7.1. Let $H$ be a positive self-adjoint operator on a Hilbert space $\mathscr{X}$ and $S$ the self-adjoint semigroup generated by $H$.

If $n \in \mathbb{N}, \gamma \in\langle 0, n\rangle$ and $p=2$ then $\mathscr{X}_{\gamma}^{n, S}=D\left(H^{\gamma}\right)$. Moreover, the norms $x \mapsto\|x\|_{\gamma}^{n, S}$ and $x \mapsto\left\|H^{\gamma} x\right\|+\|x\|$ are equivalent.

PROOF. We may assume that $\mathscr{X}=L^{2}(\mathscr{Y}, \mathscr{B}, \mu)$ for some measure space $(\mathscr{Y}, \mathscr{B}, \mu)$ and that $H$ is the operator of multiplication by a positive measurable function $h$ on $\mathscr{Y}$. Then for all $f \in L^{2}(\mathscr{Y}, \mathscr{B}, \mu)$ one has

$$
\begin{aligned}
\left(\int _ { 0 } ^ { \infty } d t t ^ { - 1 } \left(t^{-\gamma}\right.\right. & \left.\left.\left\|\left(I-S_{t}\right)^{n} f\right\|\right)^{2}\right)^{1 / 2} \\
& =\left(\int_{0}^{\infty} d t t^{-1} \int_{\mathscr{Y}} d \mu(y) t^{-2 \gamma}\left(1-e^{-t h(y)}\right)^{2 n}|f(y)|^{2}\right)^{1 / 2} \\
& =\left(\int_{0}^{\infty} d t t^{-1}\left(t^{-\gamma}\left(1-e^{-t}\right)^{n}\right)^{2}\right)^{1 / 2}\left(\int_{\mathscr{Y}} d \mu(y)\left|h(y)^{\gamma} f(y)\right|^{2}\right)^{1 / 2} \\
& =M\left\|H^{\gamma} f\right\|,
\end{aligned}
$$

where

$$
M=\left(\int_{0}^{\infty} d t t^{-1}\left(t^{-\gamma}\left(1-e^{-t}\right)^{n}\right)^{2}\right)^{1 / 2}
$$

Therefore $\mathscr{X}_{\gamma}^{n, S}=\mathscr{X}_{\gamma}^{n, S, \infty}=D\left(H^{\gamma}\right)$ and the norms are equivalent.

The $p=2$ Lipschitz norms are also equivalent with the norms $x \mapsto\left\|(I+H)^{\gamma / 2} x\right\|$ and in this form there is an obvious analogy with the fractional Sobolev norms usually defined for functions over $\mathbb{R}^{d}$ and with $H$ the Laplacian. For example, let $\mathscr{X}=L_{2}(G ; d g)$ and let $H=-\sum_{i=1}^{d} A_{i}^{2}$ be the Laplacian associated with a vector-space basis $a_{1}, \ldots, a_{d}$ of $\mathfrak{g}$ and the corresponding generators $A_{1}, \ldots, A_{d}$ of left translations $L$, that is, the operator $A_{i}$ is the generator of the one-parameter group defined by $\left(L\left(\exp \left(-t a_{i}\right)\right) f\right)(g)=f\left(\exp \left(t a_{i}\right) g\right)$. Then the spaces $D\left(H^{\gamma / 2}\right)$, equipped 
with the norms

$$
f \mapsto\|f\|_{\gamma}=\left(\int_{G} d g\left|\left((I+H)^{\gamma / 2} f\right)(g)\right|^{2}\right)^{1 / 2},
$$

are the direct generalization of the Sobolev spaces $L_{2 ; \gamma}$ normally defined for $G=\mathbb{R}^{d}$. This observation is particularly useful for illustrating the subsequent regularity results.

There are again two types of result. The first relates the action of $H$ with the spaces $\mathscr{X}_{n}^{\prime}$ and the second involves the spaces $\mathscr{X}_{n}$.

THEOREM 7.2. Let $(\mathscr{X}, G, U)$ be a unitary representation and let

$$
H=-\sum_{i, j=1}^{d^{\prime}} c_{i j} A_{i} A_{j}+\sum_{i=1}^{d^{\prime}} c_{i} A_{i}+c_{0} I
$$

where $C=\left(c_{i j}\right)$ is a real-valued strictly positive-definite matrix and $c_{0}, c_{1}, \ldots, c_{d^{\prime}} \in$ $\mathbb{C}$. Then the following are valid.

I. $\quad H$ is closed.

II. $\mathscr{X}_{\infty}$ is dense in $\mathscr{X}_{n}^{\prime}$ for all $n \in \mathbb{N}$.

Next let $S$ be the holomorphic semigroup generated by $H$.

III. There exist $\theta>0$ and $\omega>0$ such that $S$ is holomorphic in $\{z \in \mathbb{C}$ : $|\arg z|<\theta\}$ and $\left\|S_{z}\right\| \leq e^{\omega|z|}$ for all $z \in \mathbb{C}$ with $|\arg z|<\theta$.

Finally suppose $\operatorname{Re} c_{0}$ is so large that $S$ is a bounded semigroup.

IV. If $n \in \mathbb{N}$ then $\mathscr{X}_{n}^{\prime}=D\left(H^{n / 2}\right)$ and the norms $x \mapsto\|x\|_{n}^{\prime}$ and $x \mapsto$ $\left\|H^{n / 2} x\right\|+\|x\|$ are equivalent.

V. If $n>2 \gamma>0$ and $p=2$ then $D\left(H^{\gamma}\right)=\left(\mathscr{X}, \mathscr{X}_{n}^{\prime}\right)_{2 \gamma}$ and the norms $x \mapsto\left\|H^{\gamma} x\right\|+\|x\|$ and $x \mapsto\|x\|_{2 \gamma}^{(0, n)}$ are equivalent.

PROOF. We may assume that $c_{i j}=\delta_{i j}$ and that the semigroup is uniformly bounded. Let $H_{0}=-\sum_{i=1}^{d^{\prime}} A_{i}^{2}+I$. Since $H_{0}$ is symmetric and the closure $\bar{H}_{0}$ generates a semigroup $S^{(0)}$ the closure must be self-adjoint. Furthermore

$$
\left(H_{0} x, x\right)=\|x\|^{2}+\sum_{k=1}^{d^{\prime}}\left\|A_{k} x\right\|^{2} \geq\|x\|^{2}
$$

for all $x \in \mathscr{X}_{2}^{\prime}$ and hence $\bar{H}_{0}$ is strictly positive. In addition, $\|x\|_{1}^{\prime} \leq\left\|\bar{H}_{0}^{1 / 2} x\right\|$ for all $x \in \mathscr{X}_{2}^{\prime}$. Then by Lemma 7.1 and Theorems 2.1 and 3.2 there exist $c_{1}, \ldots, c_{5}>0$ 
such that for all $x \in \mathscr{X}_{3}^{\prime}$

$$
\begin{aligned}
\|x\|_{2}^{\prime} & \leq\left\|\bar{H}_{0}^{1 / 2} x\right\|+\max _{k \in\left\{1, \ldots, d^{\prime}\right\}}\left\|\bar{H}_{0}^{1 / 2} A_{k} x\right\| \\
& \leq c_{1}\|x\|_{1 / 2}^{1, S^{(0)}}+c_{1} \max _{k \in\left\{1, \ldots, d^{\prime}\right\}}\left\|A_{k} x\right\|_{1 / 2}^{1, S^{(0)}} \\
& \leq c_{2}\|x\|_{1}^{(0,2)}+c_{2} \max _{k \in\left\{1, \ldots, d^{\prime}\right\}}\left\|A_{k} x\right\|_{1}^{(0,2)} \\
& \leq 2 c_{2}\|x\|_{1 ; 1}^{(0,2)} \leq c_{3}\|x\|_{2}^{(0,3)} \leq c_{4}\|x\|_{1}^{2, S^{(0)}} \leq c_{5}\left\|\bar{H}_{0} x\right\|
\end{aligned}
$$

where the Lipschitz norms correspond to the value $p=2$. Since $\mathscr{X}_{\infty}$ is dense in $D\left(\bar{H}_{0}\right)$ and $\mathscr{X}_{2}^{\prime}$ is complete, it follows that $D\left(\bar{H}_{0}\right) \subseteq \mathscr{X}_{2}^{\prime}$. But by definition $\mathscr{X}_{2}^{\prime}=D\left(H_{0}\right) \subseteq D\left(\bar{H}_{0}\right)$. Therefore $D\left(\bar{H}_{0}\right)=\mathscr{X}_{2}^{\prime}$ and $\bar{H}_{0}=\bar{H}_{0}$. In particular, $H_{0}$ is self-adjoint.

Define $h: \mathscr{X}_{1}^{\prime} \rightarrow \mathbb{C}$ by

$$
h(x)=\|x\|^{2}+\sum_{i=1}^{d^{\prime}}\left\|A_{i} x\right\|^{2} .
$$

Then $h$ is a closed quadratic form and by the second representation theorem for quadratic forms (see [13, Theorem VI.2.23]) there exists a positive self-adjoint operator $K$ such that $D\left(K^{1 / 2}\right)=\mathscr{X}_{1}^{\prime}$ and $h(x)=\left\|K^{1 / 2} x\right\|^{2}$ for all $x \in \mathscr{X}_{1}^{\prime}$. But one straightforwardly verifies that $K$ extends $H_{0}$ and since both operators are self-adjoint it follows that $K=H_{0}$. Consequently

$$
\left\|H_{0}{ }^{1 / 2} x\right\|^{2}=\|x\|^{2}+\sum_{i=1}^{d^{\prime}}\left\|A_{i} x\right\|^{2}
$$

for all $x \in \mathscr{X}_{1}^{\prime}$. Therefore $D\left(H_{0}^{1 / 2}\right)=\mathscr{X}_{1}^{\prime}$ and the norms are equivalent.

Now let $n \in \mathbb{N}$. Then by Theorems 2.1.III and 3.2, and Lemma 7.1

$$
\begin{aligned}
\mathscr{X}_{n+1}^{\prime} & =\bigcap_{\substack{k \in\{0 \ldots, n) \\
i_{1}, \ldots, i_{k} \in\left\{1, \ldots, d^{\prime}\right\}}}\left\{x \in D\left(A_{i_{1}} \ldots A_{i_{k}}\right): A_{i_{1}} \ldots A_{i_{k}} x \in \mathscr{X}_{1}^{\prime}\right\} \\
& =\bigcap_{\substack{k \in\{0 \ldots, \ldots n) \\
i_{1}, \ldots, i_{k} \in\left[1, \ldots, d^{\prime}\right\}}}\left\{x \in D\left(A_{i_{1}} \ldots A_{i_{k}}\right): A_{i_{1}} \ldots A_{i_{k}} x \in\left(\mathscr{X}, \mathscr{X}_{2}^{\prime}\right)_{1}\right\} \\
& =\left(\mathscr{X}, \mathscr{X}_{2}^{\prime}\right)_{1 ; n}=\left(\mathscr{X}, \mathscr{X}_{n+2}^{\prime}\right)_{n+1}=\mathscr{X}_{(n+1) / 2}^{n+1, S}=D\left(H_{0}^{(n+1) / 2}\right)
\end{aligned}
$$

and the norms are equivalent. Again we have used the Lipschitz spaces with $p=2$. This proves Statements I and IV for the operator $H_{0}$ and Statement II is proved.

Equation (22) establishes $D\left(\bar{H}^{n}\right)=D\left(H_{0}^{n}\right)=\mathscr{X}_{2 n}^{\prime}$ for all $n \in \mathbb{N}$. In particular, $D(\bar{H})=\mathscr{X}_{2}^{\prime}=D(H)$ and $H$ is closed. This proves Statement I. Fix $n \in \mathbb{N}$. Then 
there exists an operator $P$ of order $2 n-1$ in the operators $A_{1}, \ldots, A_{d^{\prime}}$ such that $H^{n} x=H_{0}^{n} x+P x$ for all $x \in \mathscr{X}_{2 n}^{\prime}$. Hence

$$
\operatorname{Re}\left(H^{n} x, x\right) \geq \operatorname{Re}\left(H_{0}^{n} x, x\right)+\operatorname{Re}(P x, x) \geq \operatorname{Re}\left(H_{0}^{n} x, x\right)-|\operatorname{Re}(P x, x)| .
$$

Now $P$ is a linear combination of monomials $M_{k}$ of order $k \leq 2 n-1$ in the operators $A_{1}, \ldots, A_{d^{\prime}}$. Write $M_{k}=M_{r} M_{s}$ with $M_{r}$ and $M_{s}$ monomials of the order $r \leq n$ and $s \leq n-1$. Then

$$
\left|\left(M_{k} x, x\right)\right|=\left|\left(M_{s} x, M_{r}^{*} x\right)\right| \leq\left\|M_{s} x\right\|\left\|M_{r}^{*} x\right\| \leq\|x\|_{n-1}^{\prime}\|x\|_{n}^{\prime}
$$

for all $x \in \mathscr{X}_{2 n}^{\prime}$. So there exists $c_{1}>0$ such that

$$
|(P x, x)| \leq c_{1}\|x\|_{n-1}^{\prime}\|x\|_{n}^{\prime}
$$

for all $x \in \mathscr{X}_{2 n}^{\prime}$. For all $\varepsilon>0$ we have

$$
\|x\|_{n-1}^{\prime}\|x\|_{n}^{\prime} \leq \varepsilon\left(\|x\|_{n}^{\prime}\right)^{2}+(4 \varepsilon)^{-1}\left(\|x\|_{n-1}^{\prime}\right)^{2} .
$$

Moreover, arguing as in the proof of [22, Lemma III.3.3], there exists a $c_{2}>0$ such that

$$
\|x\|_{n-1}^{\prime} \leq \delta\|x\|_{n}^{\prime}+c_{2} \delta^{1-n}\|x\|
$$

for all $\delta \in\langle 0,1]$. Therefore setting $\delta=\varepsilon$ one obtains

$$
2|(P x, x)| \leq 3 c_{1} \varepsilon\left(\|x\|_{n}^{\prime}\right)^{2}+c_{1} c_{2}^{2} \varepsilon^{-2 n+1}\|x\|^{2}
$$

for all $x \in \mathscr{X}_{2 n}^{\prime}$. Hence for all $\lambda \geq 0$ and $x \in \mathscr{X}_{2 n}^{\prime}$

$$
\begin{aligned}
\operatorname{Re}\left(\left(H^{n}+\lambda I\right) x, x\right) & \geq \operatorname{Re}\left(H_{0}^{n} x, x\right)+\lambda\|x\|^{2}-|(P x, x)| \\
& \geq\left(p^{2}-3 c_{1} \varepsilon / 2\right)\left(\|x\|_{n}^{\prime}\right)^{2}+\left(\lambda-c_{1} c_{2}^{2} \varepsilon^{-2 n+2} / 2\right)\|x\|^{2},
\end{aligned}
$$

where $p>0$ is such that

$$
\left(H_{0}^{n} x, x\right)=\left\|H_{0}^{n / 2} x\right\|^{2} \geq\left(p\|x\|_{n}^{\prime}\right)^{2} .
$$

By choosing $\varepsilon$ small it follows that

$$
\operatorname{Re}\left(\left(H^{n}+\lambda I\right) x, x\right) \geq 0
$$

for all $x \in \mathscr{X}_{2 n}^{\prime}$ and $\lambda \geq 0$. Now $H_{0}$ and hence $H$ are generators of holomorphic semigroups with holomorphy angle $\pi / 2$. So by [21, Lemma 4.1], $H_{0}^{n}$ and $H^{n}$ are also generators of semigroups. Thus $H^{n}+\lambda I$ is a closed maximal accretive operator as is $H_{0}^{n}$. But $D\left(H^{n}\right)=D\left(H_{0}^{n}\right)$ by $(22)$ and the norms on the two spaces are equivalent. 
Then by the corollary following [12, Theorem 1] $D\left(\left(H^{n}+\lambda I\right)^{\gamma / n}\right)=D\left(H_{0}^{\gamma}\right)$ for all $\gamma \in[0, n]$ and the norms are equivalent. Therefore

$$
D\left(H^{\gamma}\right)=D\left(\left(H^{n}\right)^{\gamma / n}\right)=D\left(\left(H^{n}+\lambda I\right)^{\gamma / n}\right)=D\left(H_{0}^{\gamma}\right) .
$$

In particular, for $k \in\{1, \ldots, 2 n\} D\left(H^{k / 2}\right)=D\left(H_{0}^{k / 2}\right)=\mathscr{X}_{k}^{\prime}$ and the norms are equivalent by Statement IV for the operator $H_{0}$. Moreover, if $m>2 \gamma$ we have

$$
D\left(H^{\gamma}\right)=D\left(H_{0}^{\gamma}\right)=\mathscr{X}_{\gamma}^{m, S^{(0)}}=\left(\mathscr{X}, \mathscr{X}_{m}^{\prime}\right)_{2 \gamma}
$$

by Lemma 7.1. This proves Statements IV and V.

Finally the proof of Statement III is essentially the same as the proof of [2, Corollary 4.1]. The only difference is that the $C^{n}$-norm $\|\cdot\|_{n}$ is replaced by the subelliptic equivalent $\|\cdot\|_{n}^{\prime}$. This replacement does not affect the validity of the argument.

The second regularity result relating to the $C^{n}$-subspaces $\mathscr{X}_{n}$ is a direct corollary of previous results.

COROLlary 7.3. Adopt the assumptions of Theorem 7.2 and suppose $\operatorname{Re} c_{0}$ is large enough that $S$ is a bounded semigroup. If $n>2 \gamma>0$ and $p \in[2, \infty]$ then $D\left(H^{\gamma}\right) \subseteq\left(\mathscr{X}, \mathscr{X}_{n}\right)_{2 \gamma / r}$ and the embedding is continuous, that is, there is a $c>0$ such that

$$
\|x\|_{2 \gamma / r}^{(0, n)} \leq c\left(\left\|H^{\gamma} x\right\|+\|x\|\right)
$$

for all $x \in D\left(H^{\gamma}\right)$.

The statement for $p=\infty$ follows from Theorem 6.2 and for $p=2$ from Theorem 7.2. The result for $2<p<\infty$ then follows from the Hölder inequality.

The last statement of Theorem 6.2 is of course applicable to unitary representations and this gives more detail on the action of $H$ relative to the Lipschitz spaces.

If $a_{1}, \ldots, a_{d^{\prime}}$ is an algebraic basis with rank $r=2$ and $H$ a corresponding subelliptic operator it follows from the corollary that $\mathscr{X}_{2} \subseteq D(H) \subseteq \mathscr{X}_{1}$ and in the next example we demonstrate that this is optimal in the sense that $D(H)$ is not contained in any of the spaces that interpolate between $\mathscr{X}_{1}$ and $\mathscr{X}_{2}$. We also use the example to illustrate that $\mathscr{X}_{1}^{\prime}$ is not invariant under the representation.

EXAMPLE 7.4. Let $G$ be the Heisenberg group $A(\mathbb{R})$. So $G=\mathbb{R} \times \mathbb{R} \times \mathbb{T}$ with multiplication given by

$$
\left(a_{1}, b_{2}, z_{1}\right) \circ\left(a_{2}, b_{2}, z_{2}\right)=\left(a_{1}+a_{2}, b_{1}+b_{2}, z_{1} z_{2} e^{i a_{1} b_{2}}\right) .
$$


Next let

$$
\mathscr{X}=\bigoplus_{n=1}^{\infty} \mathscr{Y}_{n}
$$

where $\mathscr{Y}_{n}=L_{2}(\mathbb{R} ; d x)$. Then for each $(a, b, z) \in G$ and $f=\left(f_{n}\right)_{n \in \mathbb{N}} \in \mathscr{X}$ define $U(a, b, z) f \in \mathscr{X}$ by

$$
(U(a, b, z) f)_{n}(x)=z^{n} e^{i b n x} f_{n}(a+x) .
$$

It follows that $(\mathscr{X}, G, U)$ is a strongly continuous unitary representation. Let $a_{1}, a_{2}, a_{3}$ be the standard basis in the Lie algebra $g$ of $G$. Then $\left[a_{1}, a_{2}\right]=a_{3}$ and $a_{1}, a_{2}$ is an algebraic basis with rank $r=2$.

If $P$ and $Q$ are the self-adjoint operators in $L_{2}(\mathbb{R} ; d x)$ such that

$$
\begin{aligned}
& (P f)(x)=i f^{\prime}(x), \\
& (Q f)(x)=i x f(x),
\end{aligned}
$$

for all $f \in C_{c}^{\infty}(\mathbb{R})$ and $x \in \mathbb{R}$ then

$$
\begin{aligned}
& \left(A_{1} f\right)_{n}=-i P f_{n}, \\
& \left(A_{2} f\right)_{n}=i n Q f_{n}, \\
& \left(A_{3} f\right)_{n}=i n f_{n},
\end{aligned}
$$

where $A_{i}=d U\left(a_{i}\right)$. Next consider the subelliptic operator $H=-A_{1}^{2}-A_{2}^{2}$ and remark that

$$
(H f)_{n}=\left(P^{2}+n^{2} Q^{2}\right) f_{n}
$$

for all $f \in D(H)$. Let $\psi_{0}$ be the 0 th Hermite function on $L_{2}(\mathbb{R} ; d x)$ and for $n \in \mathbb{N}$ define $\varphi_{n} \in L_{2}(\mathbb{R} ; d x)$ by setting

$$
\varphi_{n}(x)=n^{1 / 4} \psi_{0}\left(n^{1 / 2} x\right)
$$

Then $\left\|\varphi_{n}\right\|=1$ and

$$
\left(P^{2}+n^{2} Q^{2}\right) \varphi_{n}=n \varphi_{n}
$$

for all $n \in \mathbb{N}$. Next let $\left(\lambda_{n}\right)_{n \in \mathbb{N}} \in l^{2}(\mathbb{N})$ and consider $f=\left(f_{n}\right)_{n \in \mathbb{N}} \in \mathscr{X}$ where $f_{n}=\lambda_{n} \varphi_{n}$. It follows from the above that $f \in D(H)$ if, and only if,

$$
\sum_{n=1}^{\infty}\left|n \lambda_{n}\right|^{2}<\infty
$$

Next let $\Delta=-A_{1}^{2}-A_{2}^{2}-A_{3}^{2}=H-A_{3}^{2}$ denote the full Laplacian. Then $\Delta \varphi_{n}=n(n+1) \varphi_{n}$. Now if $S$ denotes the semigroup generated by $\Delta$ one has 
$\left(\mathscr{X}_{1}, \mathscr{X}_{2}\right)_{\gamma}=\left(\mathscr{X}, \mathscr{X}_{2}\right)_{1+\gamma}=\mathscr{X}_{(1+\gamma) / 2}^{1, s}$ for $\gamma \in(0,1)$ and hence $f \in\left(\mathscr{X}_{1}, \mathscr{X}_{2}\right)_{\gamma}$ if, and only if,

$$
\int_{0}^{1} d t t^{-1}\left(t^{-(1+\gamma)}\left\|\left(I-S_{t}\right) f\right\|^{2}\right)^{p / 2}<\infty .
$$

Moreover, if $\varepsilon \in\langle 0, \gamma\rangle$ and $\lambda_{n}=n^{-(3+2 \varepsilon) / 2}$ then $f \in D(H)$. But if $t_{0} \in\langle 0,1 / 2\rangle$ and $n_{0}$ is an integer such that $n_{0}^{2} t_{0} \geq 1$ then

$$
\begin{aligned}
\int_{0}^{1} d t t^{-1}\left(t^{-(1+\gamma)}\left\|\left(I-S_{t}\right) f\right\|^{2}\right)^{p / 2} & \geq \int_{t_{0}}^{1} d t t^{-1}\left(t^{-(1+\gamma)} \sum_{n \geq n_{0}}\left(1-e^{-n^{2} t}\right)^{2} n^{-(3+2 \varepsilon)}\right)^{p / 2} \\
& \geq 2^{-p} \int_{t_{0}}^{1} d t t^{-1} t^{-(1+\gamma) p / 2}\left(\sum_{n \geq n_{0}} n^{-(3+2 \varepsilon)}\right)^{p / 2} \\
& \geq c_{\gamma, p}\left(n_{0}^{2} t_{0}\right)^{-(1+\varepsilon) p / 2} t_{0}^{-(\gamma-\varepsilon) p / 2}
\end{aligned}
$$

for a suitable $c_{\gamma, p}>0$ independent of $n_{0}$ and $t_{0}$. But one can choose $n_{0}, t_{0}$ such that the right hand side is arbitrarily large. Therefore $f \notin\left(\mathscr{X}_{1}, \mathscr{X}_{2}\right)_{\gamma}$. Consequently $D(H) \nsubseteq\left(\mathscr{X}_{1}, \mathscr{X}_{2}\right)_{\gamma}$ for any choice of $\gamma \in\langle 0,1\rangle$ and $p \in[1, \infty]$.

Finally $f \in \mathscr{X}_{1}^{\prime}$ if, and only if,

$$
\sum_{n=1}^{\infty}\left|n^{1 / 2} \lambda_{n}\right|^{2}<\infty
$$

But if $t>0$ and $g=\exp \left(t a_{1}\right)$ then $(U(g) f)_{n}(x)=f_{n}(x+t)$ and

$$
\left(A_{2} U(g) f\right)_{n}(x)=\operatorname{inx} f_{n}(x+t)=\operatorname{inx} x \lambda_{n} n^{1 / 4} \psi_{0}\left(n^{1 / 2}(x+t)\right) .
$$

Moreover

$$
\begin{aligned}
\int_{-\infty}^{\infty} d x\left|i n x \lambda_{n} n^{1 / 4} \psi_{0}\left(n^{1 / 2}(x+t)\right)\right|^{2} & =\int_{-\infty}^{\infty} d x\left|n^{1 / 2}\left(x-n^{1 / 2} t\right) \lambda_{n} \psi_{0}(x)\right|^{2} \\
& =\left|n^{1 / 2} \lambda_{n}\right|^{2}\left\|Q \psi_{0}-n^{1 / 2} t \psi_{0}\right\|^{2}
\end{aligned}
$$

Now $Q \psi_{0}=2^{-1 / 2} \psi_{1}$ where $\psi_{1}$ denotes the second Hermite function which is orthogonal to $\psi_{0}$. Therefore $\left\|Q \psi_{0}-n^{1 / 2} t \psi_{0}\right\|^{2}=1 / 2+\left|n^{1 / 2} t\right|^{2}$. Hence $U(g) f \in D\left(A_{2}\right)$ if, and only if,

$$
\sum_{n=1}^{\infty}\left|n^{1 / 2} \lambda_{n}\right|^{2}\left(1 / 2+\left|n^{1 / 2} t\right|^{2}\right)<\infty .
$$

Thus by appropriate choice of $\lambda_{1}, \lambda_{2}, \ldots$ one can arrange that $f \in \mathscr{X}_{1}^{\prime}$ but $U(g) f \notin$ $\mathscr{X}_{1}^{\prime}$. Consequently $\mathscr{X}_{1}^{\prime}$ is not $U$-invariant. 
One obvious application of the above results is to the representation of $G$ by left translations $L$ on $L_{2}=L_{2}(G ; d g)$. The left invariance of the Haar measure $d g$ ensures that $\left(L_{2}, G, L\right)$ is a unitary representation. The $p=2$ Lipschitz spaces $L_{2 ; y}$ then correspond to the usual fractional Sobolev spaces. A second less obvious application is to left translations $L$ on $L_{\hat{2}}=L_{2}(G ; d \hat{g})$ the square-integrable functions with respect to right invariant Haar measure. The representation $\left(L_{\hat{2}}, G, L\right)$ is not unitary unless $G$ is unimodular. Nevertheless the representation $g \mapsto \hat{L}(g)=\Delta(g)^{1 / 2} L(g) \Delta(g)^{-1 / 2}$, where $\Delta$ is the modular function, is unitary and this can be exploited to extend the embeddings et cetera of Theorem 7.2 and Corollary 7.3 to $\left(L_{\hat{2}}, G, L\right)$.

The important point is that the generator of $t \mapsto \hat{L}\left(e^{-t a_{i}}\right)$ is $\Delta^{1 / 2} A_{i} \Delta^{-1 / 2}=$ $A_{i}-\left(\beta_{i} / 2\right) I$ where $\beta_{i}=\left(A_{i} \Delta\right)(e)$. Therefore the $C^{n}$-subspaces and the subelliptic $C^{n}$-subspaces of $\left(L_{\hat{2}}, G, L\right)$ and $\left(L_{\hat{2}}, G, \hat{L}\right)$ coincide. Consequently the various interpolation and Lipschitz spaces associated with the two representations also coincide, up to equivalence of norms. Moreover, there is an invertible map from the subelliptic operators $H$ associated with $\left(L_{\hat{2}}, G, L\right)$ to the operators $\hat{H}$ associated with $\left(L_{\hat{2}}, G, \hat{L}\right)$ given by $\hat{H}=\Delta^{1 / 2} H \Delta^{-1 / 2}$. Hence the statements of Theorem 7.2 and Corollary 7.3 can be first deduced for $\left(L_{\hat{2}}, G, \hat{L}\right)$ and then translated into identical statements for $\left(L_{\hat{2}}, G, L\right)$.

Finally we note that the results of this section can be compared with Statements (a) and (d) of Theorem 16 of Rothschild and Stein. If the latter results are specialized to the representation $\left(L_{2}, G, L\right)$, or $\left(L_{\hat{2}}, G, \hat{L}\right)$, then they give a local version of Statements IV and V of Theorem 7.2 and the statement of Corollary 7.3. The Rothschild-Stein theorem is, however, stronger insofar as it extends to the representation of $G$ by left translations on the spaces $L_{p}(G ; d g)$, and $L_{p}(G ; d \hat{g})$, for $p \in\langle 1, \infty\rangle$. It is to be expected that our results also extend to this broader setting but this would probably require an amalgamation of our techniques with the parametrix methods used by Rothschild and Stein or the methods of singular integration theory.

\section{Miscellany}

We conclude this paper with some remarks on strongly elliptic operators of general order and on analytic vectors for subelliptic operators.

It follows from [22, Theorem I.5.1] that every strongly elliptic operator generates a holomorphic semigroup and these can be used to characterize the interpolation spaces $\left(\mathscr{X}, \mathscr{X}_{n}\right)_{\gamma}$. This was established for $\gamma \in\langle 0,1\rangle$ in [22] but the general result follows by the arguments of Section 3 .

THEOREM 8.1. Let $H$ be an $m$ th order strongly elliptic operator. 
I. Let $n>\gamma>0$. Then $\left(\mathscr{X}, \mathscr{X}_{n}\right)_{\gamma}=\mathscr{X}_{\gamma / m}^{n, S}$ and the norms $\|\cdot\|_{\gamma}^{(0, n)}$ and $\|\cdot\|_{\gamma / m}^{n, S}$ are equivalent, where $S$ denotes the semigroup generated by the closure of $H$.

II. The Lipschitz space $\left(\mathscr{X}, \mathscr{X}_{n}\right)_{\gamma}$ is invariant under the representation $U$ and the restriction $U_{\gamma}$ of $U$ to $\mathscr{Y}_{\gamma}=\left(\mathscr{X}, \mathscr{X}_{n}\right)_{\gamma}$ is a strongly continuous representation if $p \in[1, \infty)$. Moreover, if $H_{\gamma}$ is the associated operator in the representation $\left(\mathscr{Y}_{\gamma}, G, U_{\gamma}\right)$, then

$$
\mathscr{Y}_{\gamma ; n}=D\left(\left(H_{\gamma}+\omega I\right)^{n / m}\right)
$$

for all $n \in \mathbb{N}$ and all large $\omega>0$, with equivalent norms.

III. Let $n_{1}>\gamma_{1}>0, n_{2}>\gamma_{2}>0$ and $p \in[1, \infty)$. Let $\|\cdot\|_{\left\{\gamma_{1}, \eta_{2}\right\}}^{\left\{n_{1}, n_{2}\right\}}$ be the norm on the iterated Lipschitz space $\left(\left(\mathscr{X}, \mathscr{X}_{n_{1}}\right)_{\gamma_{1}},\left(\mathscr{X}, \mathscr{X}_{n_{1}}\right)_{\gamma_{1} ; n_{2}}\right)_{\gamma_{2}}$. Then

$$
\left(\left(\mathscr{X}, \mathscr{X}_{n_{1}}\right)_{\gamma_{1}},\left(\mathscr{X}, \mathscr{X}_{n_{1}}\right)_{\gamma_{1} ; n_{2}}\right)_{\gamma_{2}}=\left(\mathscr{X}, \mathscr{X}_{n_{1}+n_{2}}\right)_{\gamma_{1}+\gamma_{2}}
$$

and the norms $\|\cdot\|_{\left\{\gamma_{1}, \gamma_{2}\right\}}^{\left\{n_{1}, n_{2}\right\}}$ and $\|\cdot\|_{\gamma_{1}+\gamma_{2}}^{\left(0, n_{1}+n_{2}\right)}$ are equivalent.

PROOF. The proof of Statement I is a modification of the proof of Theorem 3.2. Steps 1 and 2 are independent of $H$, hence still valid. For the proof of Step 3 we may assume that there is no constant term in $H$. By [22, Theorem III.2.1] the semigroup $S$ has a kernel $K$ and

$$
\int_{G} d g K_{t}(g)=1
$$

since there is no constant term. Instead of the bounds (5) we have bounds

$$
\int_{G} d g\left|K_{t}(g)\right| e^{\rho|g|} \leq a e^{\omega\left(1+\rho^{m}\right)}
$$

valid uniformly for all $t \in\langle 0,1]$ and all $\rho \geq 0$, by [22, Corollary III.4.6], and instead of the Gaussian bounds (6) we have bounds

$$
\left|K_{t}(g)\right| \leq a t^{-d / m} e^{-b\left(|g|^{m} / t\right)^{1 /(m-1)}}
$$

valid for all $t \in\langle 0,1]$ and $g \in G$ and suitable $a, b>0$ by [22, Theorem III.4.1]. Finally, the bounds of Lemma 3.3 used in Step 4 must be replaced by the bounds

$$
\left\|S_{t} x\right\|_{n} \leq c t^{-n / m}\|x\|
$$

valid for all $t \in\langle 0,1]$ and $x \in \mathscr{X}$, for a suitable $c>0$. These bounds follow by [22, Theorem II.2.2]. This proves Statement $\mathrm{I}$.

By Corollary $3.4\left(\mathscr{X}, \mathscr{X}_{n}\right)_{\gamma}=\left(\widetilde{\mathscr{X}}, \widetilde{\mathscr{X}}_{n}\right)_{\gamma}$ where $\widetilde{\mathscr{X}}$ is the subspace on which $U$ acts strongly continuous. So we may restrict ourselves to the case that $(\mathscr{X}, G, U)$ 
is a strongly continuous representation. For the case $n=1$ the statement is proved in [22, Section II.4] and the proof for the general case is similar. The second part of Statement II can be proved by a simple modification of the proof of Theorem 4.1.II.

The proof of Statement III follows from the reiteration theorem and interpolation theorem (see [6, Theorems 3.2.20 and 3.2.23]). In Peetre's notation we have the following equalities.

$$
\begin{aligned}
\left(\left(\mathscr{X}, \mathscr{X}_{n_{1}}\right)_{\gamma_{1}},\right. & \left.\left(\mathscr{X}, \mathscr{X}_{n_{1}}\right)_{\gamma_{1} ; n_{2}}\right)_{\gamma_{2}} \\
& =\left(\left(\mathscr{X}, \mathscr{X}_{n_{1}+n_{2}}\right)_{\gamma_{1} /\left(n_{1}+n_{2}\right), p ; \mathrm{K}},\left(\mathscr{X}, \mathscr{X}_{n_{1}+n_{2}}\right)_{\left(\gamma_{1}+n_{2}\right) /\left(n_{1}+n_{2}\right), p ; \mathrm{K}}\right)_{\gamma_{2} / n_{2}, p ; \mathrm{K}} \\
& =\left(\mathscr{X}, \mathscr{X}_{n_{1}+n_{2}}\right)_{\left(\gamma_{1}+\gamma_{2}\right) /\left(n_{1}+n_{2}\right), p ; \mathrm{K}} \\
& =\left(\mathscr{X}, \mathscr{X}_{n_{1}+n_{2}}\right)_{\gamma_{1}+\gamma_{2}} .
\end{aligned}
$$

Moreover, the norms are equivalent.

Finally we consider analytic and Gevrey vectors for subelliptic operators.

Theorem 7.2.II established that the $C^{n}$-vectors for the directions of the algebraic basis are determined by the subelliptic operator $H$, that is, $\mathscr{X}_{n}^{\prime}=D\left(\bar{H}^{n / 2}\right)$ for all $n \in \mathbb{N}$ if the representation is unitary and $\bar{H}$ generates a bounded semigroup. Moreover, for any representation $U$ the space of $C^{\infty}$-vectors for $U$ is equal to the space of $C^{\infty}$-vectors for $\bar{H}$. Therefore one might hope that in some sense the subelliptic operator also determines the analytic and Gevrey vectors for $U$. To this end, let $\mathscr{X}_{\omega}^{\prime}(U)$ be the space of all $x \in \mathscr{X}_{\infty}$ such that there exist $c, t>0$, depending on $x$, such that $\|x\|_{n}^{\prime} \leq c t^{n} n$ ! for all $n \in \mathbb{N}_{0}$ and define $\mathscr{X}_{\omega}(U)$ analogously. If $\Delta$ is the full Laplacian then

$$
\mathscr{X}_{\omega}(U)=\mathscr{X}_{\omega}\left(\Delta^{1 / 2}\right)
$$

where $\mathscr{X}_{\omega}\left(\Delta^{1 / 2}\right)$ denotes the space of analytic vectors for $\Delta^{1 / 2}$ (see [20, Theorem 7.1] and for the more general case of $m$-th order strongly elliptic operators see [22, Theorem III.3.1]). The proof of $((26))$ is based on the inequality

$$
\|x\|_{2} \leq c(\|\Delta x\|+\|x\|)
$$

valid for all $x \in \mathscr{X}_{\infty}$ and the notion of analytic domination (see [9, Theorem 1.3]). The identification (26) was first established for unitary representations (see [8, Theorem 2]). Subsequently, using the fact that (27) is valid for all Lipschitz representations, the identification (26) was obtained for any representation.

Now let $H$ be a subelliptic operator as in Theorem 7.2 and assume that the semigroup generated by $\bar{H}$ is uniformly bounded. Then for unitary representations one has

$$
\|x\|_{2}^{\prime} \leq c(\|H x\|+\|x\|)
$$

for all $x \in \mathscr{X}_{\infty}$ so one might hope that in this situation one also has $\mathscr{X}_{\omega}^{\prime}(U)=$ $\mathscr{X}_{\omega}\left(\bar{H}^{1 / 2}\right)$. But this is not the situation as the next example demonstrates. 
EXAMPLE 8.2. Let $G=S U(2)$ and choose a basis $a_{1}, a_{2}, a_{3}$ of $\mathfrak{g}$ such that $\left[a_{1}, a_{2}\right]=$ $a_{3},\left[a_{2}, a_{3}\right]=a_{1}$ and $\left[a_{3}, a_{1}\right]=a_{2}$. Further define

$$
L_{+}=a_{1}-i a_{2}, \quad L_{-}=a_{1}+i a_{2}
$$

in the complex enveloping algebra of $\mathfrak{g}$. Then it is well known that for every $l \in$ $\left\{0, \frac{1}{2}, 1, \ldots\right\}$ there exists a unique irreducible unitary representation $\pi_{l}$ of $G$ in a Hilbert space $\mathscr{H}_{l}$ of dimension $2 l+1$. Moreover, there exists an orthonormal basis $e_{l,-l}, e_{l,-l+1}, \ldots e_{l, l}$ for $\mathscr{H}_{l}$ such that

$$
\begin{aligned}
d \pi_{l}\left(L_{+}\right) e_{l, m} & =\sqrt{l(l+1)-m(m+1)} e_{l, m+1}, \\
d \pi_{l}\left(L_{-}\right) e_{l, m} & =\sqrt{l(l+1)-m(m-1)} e_{l, m-1}, \\
d \pi\left(a_{3}\right) e_{l, m} & =i m e_{l, m}
\end{aligned}
$$

for all $m \in\{-l, \ldots, l\}$. Now let

$$
\mathscr{H}=\bigoplus_{l \in\left\{0, \frac{1}{2}, \ldots\right\}} \mathscr{H}_{l}
$$

and

$$
U=\bigoplus_{l \in\left\{0, \frac{1}{2}, \ldots\right\}} \pi_{l}
$$

Then $U$ is a unitary representation of $G$ in $\mathscr{H}$. Next let $A_{i}=d U\left(a_{i}\right)$ and introduce the sublaplacian $H=-A_{1}^{2}-A_{2}^{2}$. It follows that

$$
H e_{l, m}=\left(l(l+1)-m^{2}\right) e_{l, m}
$$

and hence if

$$
x=\sum_{N=0}^{\infty} e^{-N} e_{N^{2}, N^{2}}
$$

one has

$$
\left\|H^{n} x\right\|^{2}=\sum_{N=0}^{\infty} e^{-2 N} N^{4 n} \leq(4 n) ! \sum_{n=0}^{\infty} e^{-N}=c\left(\begin{array}{l}
4 n \\
2 n
\end{array}\right)(2 n) !^{2} \leq c 2^{4 n}(2 n) !^{2}
$$

for all $n \in \mathbb{N}_{0}$ where $c=e /(e-1)$. So $\left\|H^{n} x\right\| \leq \sqrt{c} 2^{2 n}(2 n)$ ! and $x \in \mathscr{X}_{\omega}\left(H^{1 / 2}\right)$.

We next argue that $x \notin \mathscr{X}_{\omega}^{\prime}(U)$. Suppose that $x \in \mathscr{X}_{\omega}^{\prime}(U)$. Then there exist $c, t>0$ such that $\|x\|_{n}^{\prime} \leq c t^{n} n$ ! for all $n \in \mathbb{N}_{0}$. Then by an elementary counting argument

$$
\left\|H^{2 N} d U\left(L_{-}\right)^{N} x\right\| \leq c 8^{N} t^{5 N}(5 N) ! \leq c t^{5 N} 2^{15 N} N !^{5}
$$


for all $N \in \mathbb{N}$. But

$$
\left\|H^{2 N} d U\left(L_{-}\right)^{N} x\right\| \geq e^{-N}\left\|H^{2 N} d U\left(L_{-}\right)^{N} e_{N^{2}, N^{2}}\right\|
$$

and

$$
d U\left(L_{-}\right)^{N} e_{N^{2}, N^{2}}=\lambda_{N} e_{N^{2}, N^{2}-N}
$$

with $\lambda_{N} \in \mathbb{R}, \lambda_{N} \geq 1$. Moreover, $H e_{N^{2}, N^{2}-N}=2 N^{3} e_{N^{2}, N^{2}-N}$. So $\left\|H^{2 N} d U\left(L_{-}\right)^{N} x\right\|$ $\geq e^{-N} N^{6 N} \geq e^{-N} N !^{6}$. But $e^{-N} N !^{6}>c t^{5 N} 2^{15 N} N !^{5}$ for large $N$ and so by contradiction one concludes that $x \notin \mathscr{X}_{\omega}^{\prime}(U)$.

The phenomenon exhibited by this example is of interest because all elliptic and subelliptic regularity properties of unitary representations are directly comparable at the $C^{n}$-level. Nevertheless the example illustrates the difference for analytic properties.

\section{Acknowledgements}

We are grateful to Professor I. Pesenson for informing us of his work on interpolation spaces and for sending us copies of his papers.

\section{References}

[1] R. Beals and P. Greiner, Calculus on Heisenberg manifolds, Ann. of Math. Stud. 119 (Princeton University Press, Princeton, 1988).

[2] O. Bratteli, F. M. Goodman, P. E. T. Jørgensen and D. W. Robinson, 'Unitary representations of Lie groups and Gårding's inequality', Proc. Amer. Math. Soc. 107 (1989), 627-632.

[3] O. Bratteli and D. W. Robinson, Operator algebras and quantum statistical mechanics, I, 2nd edition (Springer, New York, 1987).

[4] R. J. Burns, A. F. M. ter Elst and D. W. Robinson, ' $L_{p}$-regularity of subelliptic operators on Lie groups', J. Operator Theory, to appear.

[5] R. J. Burns and D. W. Robinson, 'A priori inequalities on Lie groups', Research Report SMS-02390, (The Australian National University, 1990).

[6] P. L. Butzer and H. Berens, Semi-groups of operators and approximation, Grundlehren Math. Wiss. 145 (Springer, Berlin, 1967).

[7] A. F. M. ter Elst, 'On the differential structure of principal series representations', J. Operator Theory 28 (1992), 309-320.

[8] R. Goodman, 'Analytic domination by fractional powers of a positive operator', J. Funct. Anal. 3 (1969), 246-264.

[9] R. Goodman and N. R. Wallach, 'Whittaker vectors and conical vectors', J. Funct. Anal. 39 (1980), 199-279.

[10] D. S. Jerison and A. Sánchez-Calle, 'Estimates for the heat kernel for a sum of squares of vector fields', Indiana Univ. Math. J. 35 (1986), 835-854. 
[11] P. E. T. Jørgensen, 'Representations of differential operators on a Lie group', J. Funct. Anal. 20 (1975), 105-135.

[12] T. Kato, 'A generalization of the Heinz inequality', Proc. Japan Acad. Ser. A Math. Sci. 37 (1961), 305-308.

[13] - Perturbation theory for linear operators, Grundlehren Math. Wiss. 132, 2nd edition (Springer-Verlag, Berlin, 1984).

[14] A. Nagel, E. M. Stein and S. Wainger, 'Balls and metrics defined by vector fields I: basic properties', Acta Math. 155 (1985), 103-147.

[15] E. Nelson, 'Analytic vectors', Ann. of Math. (2) 70 (1959), 572-615.

[16] I. Z. Pesenson, 'On interpolation spaces on Lie groups', Soviet Math. Dokl. 20 (1979), 611-616.

[17] — , 'Nikol'skiî-Besov spaces connected with representations of Lie groups', Soviet Math. Dokl. 28 (1983), 577-581.

[18] _ ' 'On an abstract theory of Nikol'skiǐ-Besov spaces', Soviet Math. (Iz. VUZ) 32 (1988), 80-92. Izv. Vyssh. Uchebn. Zaved. Mat. 32, (1988), 59-68.

[19] N.S. Poulsen, 'On $C^{\infty}$-vectors and intertwining bilinear forms for representations of Lie groups', J. Funct. Anal. 9 (1972), 87-120.

[20] D. W. Robinson, 'Lie groups and Lipschitz spaces', Duke Math. J. 57 (1988), 357-395.

[21] —_, 'Commutators and generators II', Math. Scand. 64 (1989), 87-108.

[22] __ Elliptic operators and Lie groups, Oxford Math. Monographs (Oxford University Press, Oxford, 1991).

[23] L. P. Rothschild and E. M. Stein, 'Hypoelliptic differential operators and nilpotent groups', Acta Math. 137 (1976), 247-320.

[24] E. M. Stein, Singular integrals and differential properties of functions, Princeton Math. Ser. 30 (Princeton University Press, Princeton, 1970).

[25] H. Triebel, Interpolation theory, function spaces, differential operators (North-Holland, Amsterdam, 1978).

[26] N. T. Varopoulos, 'Analysis on Lie groups', J. Funct. Anal. 76 (1988), 346-410.

[27] N. T. Varopoulos, L. Saloff-Coste and T. Coulhon, Analysis and geometry on groups, Cambridge Tracts in Mathematics 100 (Cambridge University Press, Cambridge, 1992).

[28] A. Zygmund, Trigonometric series II, 2nd edition (Cambridge University Press, Cambridge, 1959).

Centre for Mathematics and its Applications

School of Mathematical Sciences

Australian National University

ACT 0200

Australia 